\title{
The effect of focus prominence on phrasing
}

\author{
Michael Wagner, Michael McAuliffe ${ }^{* *}$ \\ Department of Linguistics, McGill University \\ 1085 Doctor Penfield Avenue, Montréal, Québec, H3A 1A7 \\ Canada
}

\begin{abstract}
Prosody simultaneously encodes different kinds of information about an utterance, including the type of speech act (which, in English, often affects the choice of intonational tune), the syntactic constituent structure (which mainly affects prosodic phrasing), and the location of semantic focus (which mainly affects the relative prosodic prominence between words). The syntactic and semantic functional dimensions (speech act, constituency, focus) are orthogonal to each other, but to which extent their prosodic correlates are remains controversial. This paper reports on a production experiment that crosses these three dimensions to look for interactions, concentrating on interactions between focus prominence and phrasing. The results provide evidence that interactions are more limited than many current theories of sentence prosody would predict, and support a theory that keeps different prosodic dimensions representationally separate.
\end{abstract}

Keywords:

prosody, prominence, focus, phrasing, intonation, deaccenting

\footnotetext{
* Principal corresponding author

** Corresponding author

Email addresses: chael@mcgill.ca (Michael Wagner), michael.mcauliffe@mail.mcgill.ca (Michael McAuliffe)
} 


\section{Introduction}

Sentence prosody encodes several different types of information, which are in principle orthogonal to each other. A speaker can make independent decisions about the speech act that they want to perform (which depends on their conversational goals), about the constituent structure of their utterance (which depends on the message they want to encode), and about which part of their utterance they want to highlight (which is influenced by what's new and what's given in the context). These decisions will affect the choice of intonational tune, prosodic phrasing, and prosodic prominence. However, the functional dimensions may not cleanly map in a one-to-one fashion to these prosodic dimensions. For example, many current accounts of how focus affects prosody in English assume that both prosodic prominence and prosodic phrasing are affected by it. More generally, theories of sentence prosody vary in the types of interactions between the dimensions they implicitly or explicitly posit.

One idea of how different syntactic and semantic factors can simultaneously affect sentence prosody is that they each independently influence the prosodic pattern of an utterance in an additive way. This is essentially the assumption in socalled 'overlay' models (Öhmann, 1967; Fujisaki, 1981; Möbius, 1993; Bailly and Holm, 2005; Xu, 2005; Gerazov et al., 2018), in which separate functions com-

pose to form the overall acoustic contour of a sentence. For example, Öhmann (1967) argues that the choice of lexical pitch accent on a word and the choice of intonational tune in a sentence each independently affect the $\mathrm{F}_{0}$-contour of utterances in Swedish. $\mathrm{Xu}(2005)$ argues that focus and grouping can independently affect the $\mathrm{F}_{0}$ in addition, as illustrated in Fig. 1. Overlay models are in principle compatible with interactions. However, there is no prediction what these interac- 
tions should be. For example, they make no predictions about whether and how a change in the location of focus (the 'focal' function in Fig. 1) and the prosodic reflexes of constituent structure (the the 'grouping' function in Fig. 1) should interact with each other.

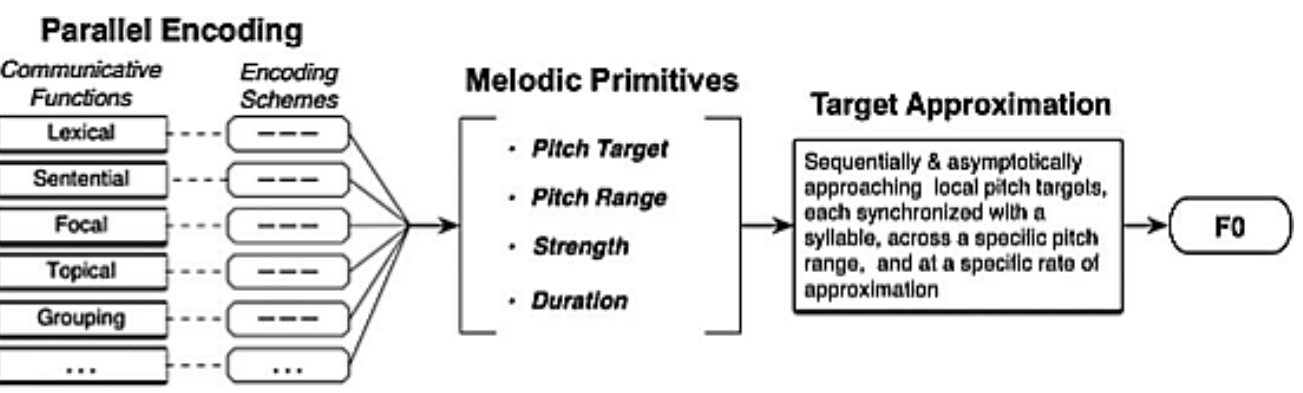

Figure 1: The Penta model: Parallel encoding of different functions in the speech signal (from $\mathrm{Xu}$. 2005)

A very different conception of how these factors combine to shape the overall prosody of an utterance is assumed in most phonological models of sentence prosody (e.g. Pierrehumbert, 1980; Ladd, 1980; Selkirk, 1984), and embodied in the ToBI transcription of North American English (Beckman and Ayers Elam, 1997; Beckman et al. 2005). These accounts are often referred to as the Autosegmental-Metrical Model of sentence prosody ('AM-Model', cf. Ladd, 2008). They are based on a metrical representation that aligns with the segments of an utterance but is in principle independent of it. While in overlay models, the functional dimensions, for example syntactic constituent structure, directly influence the output signal, they do so only indirectly in AM-models. The metrical representation is assumed to consist of a hierarchy of phonological constituents. An example of such a prosodic hierarchy from Selkirk $(1986,384)$ is given in Fig. 2 (we added the Greek letters that are often used to refer to each level, see Féry 
2013). Note that the 'phonological phrase' is often called 'intermediate phrase' instead, e.g. in the ToBI model of American English.

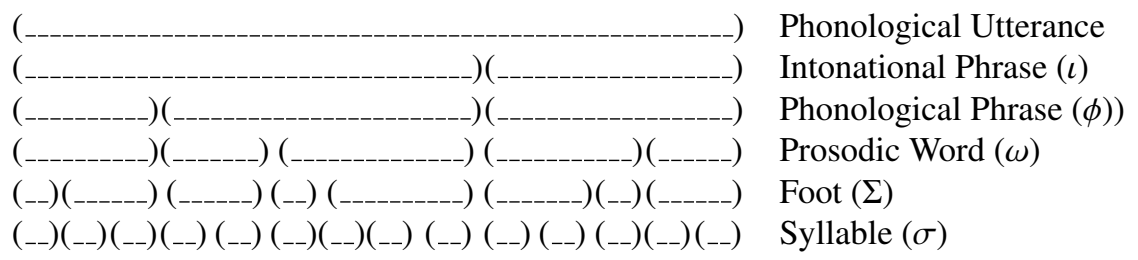

Figure 2: The Prosodic Hierarchy according to Selkirk (1986, 384)

In this model, various factors influence the precise prosodic structure. The effect of syntactic constituent structure, for example, may affect the location of phonological and intonational phrase boundaries. Existing phonological models of sentence prosody implicitly or explicitly 'build in' specific assumptions about interactions between the different factors influencing sentence prosody. For example, focus is often assumed to influence not just prominence, but also phrasing. The idea is that post-focal material remains unaccented and is 'dephrased' (a term from Jun and Fougeron 2000), meaning that it becomes part of the intonational phrase along with the preceding focused material.

This idea was already implicit in the model of English intonation proposed in Pierrehumbert (1980). For example, Pierrehumbert (1980, 265) observes that the question in (1a), with neutral focus, often contains an intonational break between the subject and the VP, as indicated by the percent sign in the example. This break effectively signals that the material within the VP forms a constituent to the exclusion of the subject. This boundary seems to vanish, however, when prominence is shifted to the subject, as in (1b), where the entire question is mapped to a single intonational and intermediate phrase. The rise for the intonational phrase bound- 
ary is realized at the end, and the rise for the intermediate phrase boundary starts right after the low pitch on Manitowoc. This rendition of the question with main prominence on Manitowoc would be adequate as a way to challenge someone else's contextually salient claim that Every small town has a library:
a. Does Manitowoc \% have a library?
b. Does Manitowoc have a library?

The reason behind the assumption that phrasing in the post-focal domain must be erased is that certain categories of phrases (such as intonational or intermediate phrases) are assumed to necessarily require tonal events--which, the assumption is, cannot be realized post-focally. In general, it should not be possible then to realize phrasing distinctions within the VP if focus is placed on the subject, since the tonal events that cue these phrasing distinctions are not available in an unaccented stretch. The assumption that phrasing is necessarily cued by tonal events is fairly standard in the AM literature. Beckman (1996), for example, argues that "[...] there must be at least one pitch accent somewhere in every (prosodic) phrase $[\ldots]$ ". Hence in the post-focal domain, where pitch accents are typically absent, phrasing distinctions should be neutralized.

The question of whether and how focus-induced prominence effects interact with the prosodic phrasing remains central in deciding between current theories of sentence prosody, and is the main research question of this paper. In an earlier test of the claim that early focus obliterates later phrasing distinctions, Norcliffe and Jaeger (2005) found that durational cues for phrasing in the post-focal part of sentences remain intact. More empirical evidence against (complete) post-focal dephrasing in a variety of languages can be found in Hayes and Lahiri (1991), Jun 
and Fougeron (2000), Sugahara (2003), Ishihara (2003), Ishihara (2007), Féry (2010), Jun (2011), Ishihara (2016) and Kügler and Féry (2017). If true, this is as expected in overlay approaches, and are also compatible with phonological models that do not conflate focal prominence and phrasing. More generally, how various functional dimensions interact can decide between different models of sentence prosody.

In the following section, we report on prior findings regarding the prosodic realization of the different functional dimensions (speech act, constituency, focus). In section 3, we report on a production experiment with a factorial design that crosses the three functional dimensions in order to see how they interact in their prosodic effects. Section 4 will describe an additional analysis using an automatic classification with random forests, which will contribute further insights. We conclude with a discussion of the theoretical implications of our findings for the phonological representation of sentence prosody.

\section{Three Dimensions}

Focus, syntactic constituent structure, and type of speech act each affect sentence prosody in systematic ways (Ladd, 2008). In this section we will review how the three functional dimensions affect prosodic prominence, prosodic phrasing, and choice of intonational tune, and also point to some potential interactions.

\subsection{Focus and Prosodic Prominence}

In English and many other languages, the prosody of an utterance can be affected by whether a sub-constituent is marked as contextually contrastive or as contextually given (see Krifka, 2008, for a review of these notions). 
(2) A: Jane dove into the water.

B: \#Then Dillon dove into the water.

B': Then Dillon dove into the water.

In A's initial assertion, both the words Jane and water typically would carry a pitch accent. In the response to A, however, the VP cannot include a clear and salient accent on water, and prominence has to shift to the subject Dillon, as in B's response. How can we explain this?

Could we simply say Dillon receives a contrastive accent in (2) because it contrasts with Jane, and that this somehow obliterates accents later in the utterance? This characterization would not be sufficient. In the following exchange, there would be no focus prosody in B's utterance, although the two noun phrases, Jane and Dillon, contrast here as well:
A: Jane sat down.
B: \#Then Dillon dove into the water.
B' Then Dillon dove into the water.

The crucial difference to the prior dialogue is that dove into the water is not previously mentioned here. Could we say instead then that for the prominence shift to obtain, dove into the water has to be contextually given? This also seems insufficient:

(4) A: Dillon dove into the water?

B: Dillon dove into the water.

$\mathrm{B}^{\prime}$ \#Dillon dove into the water. 
While repeating the whole sentence may not be the preferred way to convey a positive response here, it is certainly a possible one. However, placing focus prominence on Dillon and reducing VP is impossible here, although the VP is contextually given. It is possible only if B wants to evoke a contrast to somebody other than Dillon who did or did not jump into the water. The fact that we infer that such a contrast is evidence that focus prominence on Dillon in fact requires a contrast to Dillon as well as requiring that the VP is contextually given. The correct generalization is that this prosody requires a contrasting alternative of the form $x$ dove into the water.

So in order to state the correct generalization about focus prosody, we need to refer both to what's contrastive (the material that is substituted in the antecedent), and to what's given (the material that is already present in the antecedent). And both contrastive and given material is acoustically affected by focus: Contrastive material is prosodically boosted, given material prosodically reduced (Eady and Cooper, 1986, Breen et al., 2010, i.a.) 1 $^{1}$

Rooth (1985, 1992), building on ideas in Chomsky (1971) and Jackendoff (1972), proposed an influential theory of how to account for such focus and givenness effects. At the heart of this account is the notion that each constituent, in addition to its semantic denotation, comes with a set of alternative meanings (in the trivial case, just the meaning of the constituent itself). A second ingredient of this theory is the syntactic operator $\sim$, which can attach to any constituent and operates over its alternatives. It introduces the presupposition that a set of alternatives

\footnotetext{
${ }^{1}$ While the arguments given here are hardly sufficient to show this conclusively, see Klassen and Wagner (2017) for a series of experiments that support the conclusion that prominence shifts necessarily require contrastive alternatives.
} 
to this constituent is contextually salient. The antecedent alternatives have to be identical to the constituent $\sim$ attaches to, modulo substitutions of constituents that bear a syntactic F-marker. In this theory, B's response in (2) would be represented as follows: Then $\left[[\text { Dillon }]_{F}\right.$ dove into the water $] \sim$

For the presupposition of $\sim$ to be satisfied in (5), there must be a contextually salient set of semantic alternatives of the form $x$ dove into the water, where $x$ stands in for one or more contextually relevant alternatives to Dillon. One can characterize the prosodic effect of $\sim$ as follows: F-marked material within the scope of $\sim$ ('material marked as focused') is prosodically boosted, while non-Fmarked material in the scope of $\sim$ ('material marked as given') is prosodically reduced (cf. Rooth 1992; Truckenbrodt 1995). This can be thought of as a constraint on the relative prosodic prominence between constituents: Focused constituents need to be more prominent than given constituents (cf. Williams, 1997; Szendröi, 2003; Wagner, 2005; Reinhart, 2006; Büring, 2016). The focus presupposition in (2), for example, is encoded by flipping the relative prominence relation between subject and VP. Whereas by default, the VP must be more prominent than the subject, the focus structure in (2) has the effect that the subject ends up more prominent than the VP.

This notion of 'relative prominence' is of course not an innocent assumption. An important question for prominence-based approaches to focus-marking is whether the notion of relative prominence is directly entwined with the notion of prosodic phrasing, which also affects perceptual prominence. Our data will provide new insights on this question, which we will discuss in detail the final 
section of the paper. $2^{2}$

Prior studies have shown that the phonetic realization of focus prominence in English involves an increase in duration, maximal pitch, pitch range, and intensity of the stressed syllable of the focused constituent, and a reduction in duration, pitch range, and intensity on the material following the focused constituent (Cooper et al., 1985; Eady and Cooper, 1986; Breen et al., 2010, i.a.). The pitch range reduction has been reported to manifest itself differently depending on the intonational tune of an utterance. In a declarative utterance, pitch usually drops right after the accented syllable of the focused constituent and remains low throughout the post-focal domain. In an utterance with a rising question intonation, pitch usually rises right after the focused constituent and remains high throughout the entire post-focal domain.

The phonological interpretation of this post-focal reduction varies in the literature. While prior analyses often assume that post-focal constituents are completely deaccented and without any tonal targets, there are several reports of pitch movements in the post-nuclear domain, starting with Pierrehumbert $(1980,223)$, who observed post-nuclear 'echo accents.' Findings of this type have lead some authors to posit that post-focal reduction does not obliterate pitch targets altogether, but rather, it may simply compress pitch events to a more narrow range

\footnotetext{
${ }^{2}$ There are different accounts of how the phonological reflexes of focus marking that do not require a notion of relative prominence. Schwarzschild (1999), for example, argues that all and only F-marked words carry a pitch accent. The assumption fails to explain asymmetries between the last (or 'nuclear') accent of an utterance and prior accents. Welby (2003) found that the presence of prenuclear accents do not come with the interpretive effects of focus, while the presence of the nuclear accent does. These asymmetries make sense if relative prominence is what's crucial, and if, other things being equal, final constituents are more prominent than earlier constituents. We return to this below. Another weakness of this account is that it fails to explain how focus can still be encoded in the absence of any pitch accents, such as in instances of second-occurrence focus (Büring, 2016; Baumann, 2016).
} 
(e.g. Sugahara, 2003; Ishihara, 2003, 2016, Kügler and Féry, 2017). Another interpretation of at least some post-nuclear tonal events are that they are the reflex of (intermediate) phrase-accents, and align with metrically prominent syllables (see Grice et al., 2000, for a detailed discussion of phrase accents).

Our main research question here is how focus prominence affects prosodic phrasing, and more specifically whether the cues to phrasing are obliterated, or at least diminished, in the post-focal domain. In order to better understand why such an effect might be expected, let's look at how prosodic phrasing is assumed to come about.

\subsection{Syntactic constituent Structure and Phrasing}

Syntactic constituent structure affects the durational pattern of utterances, such that prosodic domains (such as the phonological phrase or the intonational phrase) often comprise words that form syntactic constituents and exclude words that are not part of the constituent. Hence, prosodic phrasing often encodes syntactic constituent structure. Lehiste (1973) demonstrated this convincingly in a study looking at whether globally ambiguous sentences are acoustically differentiated when said aloud. While some structural ambiguities were not reliably disambiguated (e.g., PP attachment ambiguities), others were. An example are coordinate structures:

(6) a. (Steve or Sam) and Bob will come.

b. Steve or (Sam and Bob) will come.

One central finding was that the length of the underlined words of these coordinate structures was increased if they contained a syntactic constituent boundary 
(marked with a parenthesis here). There are multiple durational lengthening effects that contribute to these differences. Klatt (1975) found that syllables that occur at the end of syntactic constituents are substantially lengthened. Many studies since have supported the presence of domain-final (or pre-boundary) lengthening in English and other languages (Wightman et al., 1992; Price et al., 1991; Shattuck-Hufnagel and Turk, 1996, Byrd and Saltzman, 1998; Cho and Keating, 2001; Byrd and Saltzman, 2003, Cho, 2016). In the coordinate structure in (6a), Sam would be longer compared to the strucure in $(6 \mathrm{~b})$ because it occurs at the end of a larger syntactic unit. Turk and Shattuck-Hufnagel (2007) found the boundaryinduced lengthening affects both the final syllable before the boundary as well as the last accented syllable preceding it.

Wagner (2005) investigated durational effects in coordinate structures in more detail, and found that there is also a speed-up in words that occur at the beginning of a phrase, compared to the durations observed in a simple list structure without internal syntactic branching. A second finding of interest was that the degree of pre-boundary lengthening induced by syntactic junctures within an utterance is often much greater than the final lengthening observed at the end of an utterance. In coordinate structures similar to (6), the durational difference in the final words in coordinate structure (in our example the name $\mathrm{Bob}$ ) did not show a high variation depending on its position in the structure, and generally showed a duration more comparable to utterance-internal words that were not phrase-final.

The distribution of pauses provides a further cue to syntactic grouping. Pauses have been reported as correlating less well with syntax than duration (Gollrad, 2013), probably because they can also be due to other cognitive factors, such as lexical retrieval or other types of processing difficulty (Goldman-Eisler, 1972). 
Grosjean and Collins (1979) conducted an experiment in which participants read sentences at different speech rates. They added the average pause-length between each set of two adjacent words. They could then derive a hierarchical tree structure of these sentences based on the phonetic closeness of pairs of adjacent words. These trees closely matched participants' intuition about how they would group these words. Gee and Grosjean (1983) propose a prosodic interpretation of these effects, such that the length of a pause between two words can be a cue to whether and to what extent they phrase together prosodically. Within the ToBI system of intonation, pauses are viewed as evidence as to whether there is an intonational phrase break (high likelihood of a pause) or an intermediate phrase break (lower but non-zero chance of a pause). It also includes a category (break-index 2) which is used when either a pause is present but a tonal boundary signal is absent, or when a tonal boundary signal is present but the disjuncture sounds 'weaker than expected' for an intermediate or intonational phrase break (Beckman and Ayers Elam, 1997, 35). There is some evidence that pre-boundary lengthening and pauses are perceptually integrated into a single percept of juncture. Martin (1970. 1971) found that listeners report hearing pauses although there were no unfilled pauses in the signal when there was syllable lengthening just before the perceived juncture.

Apart from final (or pre-boundary) lengthening and pause placement, phrasing has additional durational effects due to domain-initial strengthening (Fougeron and Keating, 1997; Lavoie, 2001; Cho, 2002, Keating et al., 2003; Keating, 2006; Cho et al. 2007, 2011; Cho, 2016). Domain-initial strengthening occurs at the beginning of prosodic domains, and the degree of strengthening increases cumulatively with the strength of the prosodic boundary. Fougeron and Keating (1997) 
investigated initial strengthening in arithmetic expressions:

$$
(89+89+89) * 89=\text { a lot }
$$

Participants were recorded using so-called 'reiterant' speech, which mimics the structure of such arithmetic expressions:

(nonono no nonono no nonono) no nonono equals a lot

The results showed that nasal onsets were realized with more linguo-palatal contact at the beginning of larger prosodic phrases, which also resulted in a longer duration of these nasals. Similar results were reported from an acoustic study of VOT in Korean in Jun (1993). Keating et al. (2003) reviews cross-linguistic evidence showing that such initial strengthening effects are pervasive.

Apart from durational cues, there are also $\mathrm{F}_{0}$ cues to phrasing. The most important reason why $\mathrm{F}_{0}$ often provides cues to phrasing are boundary tones (Pierrehumbert, 1980; Ladd, 2008, i.a.). Interestingly, Gollrad (2013) found in a series of production and perception experiments that $\mathrm{F}_{0}$ related cues to boundaries are important when conveying intonational phrasing, but less important when conveying (smaller) phonological phrase boundaries.

In addition to affecting boundary-tone placement, prosodic phrasing has also been reported to affect the relative scaling of pitch accents (Ladd, 1988). These scaling effects are often attributed to an adjustment of a reference line for pitch that is sensitive to prosodic phrasing. The idea is that individual tonal targets are scaled relative to this reference line. Ladd (1988) coordinated sentences as a way to probe for pitch scaling effects and found clear scaling effects between intonational phrases: 
(9) a. Ryan has a lot more money, (but Warren is a stronger campaigner and Allen has more popular policies).

b. (Ryan has a lot more money and Warren is a stronger campaigner), but Allen has more popular policies.

Ladd (1988) reports that in structures of the type in example (9a) the pitch level on the last conjunct (Allen has more popular policies) was down-steppped relative to the conjunct that precedes it (Warren is a stronger campaigner), which in turn is down-stepped relative to the first (Ryan has a lot more money). In example(9b), on the other hand, the pitch-level in both the second and third conjunct are downstepped relative to the first. Ladd takes these scaling differences to motivate a hierarchically organized metrical representation with recursively nested intonational phrases. van den Berg et al. (1992) show similar scaling effects in coordinate structures involving place names. More evidence for pitch scaling is discussed in Kubozono (1989), Kubozono (1992), van den Berg et al. (1992), Féry and Truckenbrodt (2005), Féry and Kentner (2010), Kentner and Féry (2013), Truckenbrodt and Féry (2015), and Petrone et al. (2017).

Intensity is often not considered as a cue to phrasing. For example Price et al. (1991) and Wightman et al. (1992) explore pitch and durational cues, but make no reference to intensity. Streeter (1978) considered amplitude as a cue for boundary perception, but concluded it is only of minor importance.

One reason why intensity is often disregarded may be that existing correlations between phrasing and intensity could be chalked up as an automatic consequence of exhalation, rather than an actively controlled cue. A drop in intensity over the course of an utterance is expected because exhaling reduces sub-glottal pressure (Björklund and Sundberg, 2016). There are indeed many reports that intensity 
tends to decrease throughout an utterance. Pierrehumbert (1979) reports experimental evidence for such a down-drift, and also that it interacts with the perception of prominence in perception: Failing to decrease intensity on later constituents provides a cue for prominence.

There is some indication, however, that speakers might actively control intensity in order to cue prosodic phrasing. Trouvain et al.(1998) provides an example of how intensity can be used as a cue for a phrase break in English. In a production study on prosodic phrasing in German, Poschmann and Wagner (2016) found that intensity is a cue to phrasing comparable in reliability to pre-boundary lengthening, such that intensity is adjusted high at the beginnings of phrases and then drops toward the end of the phrase. This was found to be a cue for phrasing distinctions even utterance-medially, so the scaling of intensity did not appear to be simply a result of the drop in sub-glottal pressure while exhaling.

One important aspect of intensity in speech is that it closely correlates with pitch. For example, speakers raise $F_{0}$ when aiming to talk louder for about a half semi-tone per $\mathrm{db}$ (Gramming et al., 1988). See also the discussion in Vaissière (1983). But this is not a physical necessity, speakers can in principle also talk louder while holding pitch constant. The heightened air-flow may just naturally increase the frequency of vocal fold vibration unless a speaker counter-acts that tendency. This raises the possibility that some variability of pitch due to phrasing (such as pitch-accent scaling) may actually be a passive consequence of an active manipulation of intensity by the speaker, or may just be used to enhance the primary cue of intensity. Our results support the conclusion that intensity is an important cue to phrasing in English. 


\subsection{Speech act and Intonational Tune}

Intonational tunes vary depending on the speech act that an utterance performs. The choice of intonational tune is typically assumed to affect only the tonal events of an utterance. For the present study, only declarative falls and the rises frequently used in polar questions are relevant. One distinguishing feature between these two tunes is that they end in different boundary tones, an L\% in the case of the declarative fall and an $\mathrm{H} \%$ in the case of interrogative rises. The interrogative rise is typically used in polar questions, it can also be used in wh-questions (Hedberg et al., 2014); the declarative fall is typically used in declarative assertions and wh-questions, but sometimes also in polar questions. The semantic differences between these tunes remains an open research question (see Truckenbrodt, 2012, for a review).

The rise in polar questions and the final fall in declaratives often seems to start right after the pitch-accented syllable, rather than at the end of a phrase or the utterance. This is often attributed to there being 'phrase accents' in addition to the final boundary tones as part of these tunes, following (Pierrehumbert, 1980). These are today usually taken to be boundary tones of intermediate phrases, even though they are realized right after the pitch-accented syllable (see Grice et al. 2000, for a review and empirical evidence in favor of phrase accents), but see Barnes et al.2010 for a different interpretation).

Finally, the two tunes also differ in the pitch accents that are placed on accents words within an utterance. The English question rise is usually used with $\mathrm{L}^{*}$ pitch accents, while the English declaratives fall usually with $\mathrm{H}^{*}$ or $\mathrm{H}^{*} \mathrm{~L}$ pitch accents, although other combinations are possible (Pierrehumbert and Hirschberg, 1990). Duration and intensity may also be cues to differentiate different intona- 
tional tunes, but they are often not considered-our results will show they, too, can distinguish different contours.

\subsection{Interactions}

The central research question of this paper is how cues to focus prominence and cues to prosodic phrasing interact. More specifically, we are interested in the question of whether prosodic phrasing remains intact in the post-focal domain. There is some prior work that suggests that at least durational cues remain intact postfocally in English (Norcliffe and Jaeger, 2005) (see Jun and Fougeron, 2000, Sugahara, 2003; Ishihara, 2003, 2016; Kügler and Féry, 2017, for relevant results in other languages). The question of whether pitch cues to phrasing are used in the post-focal domain is still unresolved, although there are studies that suggest that the often-made claim that there are no tonal targets in the post-focal domain might not be accurate at least in some languages (Xu, 2005; Kügler and Féry, 2017). It is possible that post-nuclear tonal perturbations are due to phrase accents that do not constitute full pitch accents-we will return to this issue in the final discussion.

While prosodic phrasing, just as focus prominence, affects duration, intensity, and $\mathrm{F}_{0}$, among other cues, their precise effects differ phonetically in where exactly in the signal they occur (Edwards et al., 1991; Beckman and Edwards, 1992; Cho and Keating, 2009; Cho et al., 2011). For example, phrase-final lengthening mostly affects the final syllable, while lengthening due to prominence mostly affects the accented syllable. And yet the effects of accentual lengthening spill over into following unstressed syllables (Turk and Sawusch, 1997) and final lengthening can also affect the accented syllable (Turk and Shattuck-Hufnagel, 2007). There are also good reasons to think that prosodic phrasing might be affected by focus more directly. In many languages, focus appears to affect phrasing decisions 
(Féry, 2013, and references therein). Conversely, phrasing also seems to affect our percept of prominence. In Lehiste's coordination examples in (6), Sam seems intuitively more prominent when at the end of a prosodic phrase as in (a) than at the beginning, as in (b). Various models have claimed that this is why focus affects phrasing: foci like to align with the edges of prosodic phrases because it makes them more prominent (Truckenbrodt, 1999; Büring, 2010b). This idea fits well with the Roothian prominence-based approach to focus (but see Féry, 2013, for a different perspective).

We are also interested in other types of interactions. The choice of intonational tune, which is affected by the type of speech act, might also interact with how phrasing is encoded. For example, utterances with question rises might have very different phrasing-related pitch accent scaling effects. The question whether and how pitch-accent scaling is used in questions has also not been previously explored, as far as we know, although there is work on the realization of prosodic boundaries in questions as opposed to declaratives. ${ }^{3}$ There could also be interactions between the choice of tune and the marking of focus. According to Rooth's theory of focus, the choice of intonational tune should in principle be orthogonal to the prosodic effects of focus. But recently, such interactions have been found: (Goodhue et al., 2016) found that focus prominence is almost never used when the Contradiction Contour is chosen, but in the same context is used very frequently when a declarative fall is used. Schlöder (2018) argues that focus prominence is used differently with the rise-fall-rise contour.

\footnotetext{
${ }^{3}$ A reviewer pointed us to Feldhausen (2016), who found that in Spanish, while dislocated topics typically end with a rise in declaratives, in questions they often end with a fall and are obligatorily followed by a pause.
} 
Finally, while the role of intensity is often considered in studies of focus prominence, its role in cueing phrasing or intonation has not been given much consideration in the literature. While many prior studies have looked at the role of intensity as a cue for prosodic prominence, we are not aware of work that looks at how this interacts with the choice of tune or phrasing.

Having reviewed some key findings about cues to all three dimensions, and some particular claims about interactions between them, we can now turn to our experiment. The study crosses all three functional dimensions and aims at establishing how independently or interactively they affect the output prosody.

\section{The Experiment}

By crossing the three functional dimensions (speech act, focus, constituent structure), we will be able to see their individual phonetic import on the signal, as well as whether they interact with each other. Prior studies have usually only manipulated one dimension at a time, or at most two. Eady and Cooper (1986), in their classic study on sentence prosody, looked at focus and type of speech act (question vs. declarative), for example. We are interested here in whether the contribution of all three functional dimensions can be successfully retrieved from the acoustics, or whether the effect of one dimension sometimes neutralizes the effects of the other. Of special interest is the question of whether acoustic cues to phrasing remain intact in the post-focal domain.

\subsection{Speech materials}

For our manipulation of constituent structure, we used coordinated names (following Lehiste, 1973; van den Berg et al., 1992; Wagner, 2005; Féry and Kentner. 2010; Kentner and Féry, 2013; Petrone et al., 2017). The intended syntac- 
tic constituent structure was indicated by the placement of commas. (10) illustrates an example with left-branching ([AB]C), and (11) illustrates right-branching $(\mathrm{A}[\mathrm{BC}]): 4$

Declarative, Focus on Conjunct B, Left-Branching:

You said that Megan and Dillon, or Morgan would help. But in fact we were told that $\operatorname{Megan}_{A}$ and Lauren $B$, or Morgan $C$ would help.

Declarative, Focus on Conjunct B, Right-Branching:

You said that Megan, and Dillon or Morgan would help. But in fact we were told that Megan $_{A}$, and Lauren or Morgan $_{C}$ would help.

We varied the type of speech act simply by using a period at the end of the target utterance as in (10) and (11), indicating that it was intended as an assertion, or alternatively a question mark (12), indicating that it was intended as a polar question. We expected that participants would produce a rising intonation for questions, since this is the canonical intonation for polar questions in American English (Ladd, 2008; Hedberg et al., 2010) ${ }^{5}$ Interrogative, Broad Focus, Left-Branching:

\footnotetext{
${ }^{4}$ See the supplementary materials for a complete list of the stimuli.

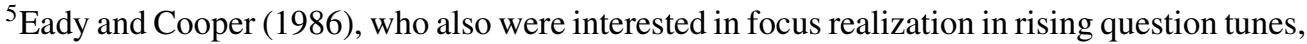
looked at declaratives vs. wh-questions instead. This seems like a risky choice, however. Most polar questions in American English are realized with a rising intonation, and especially those with declarative syntax are very likely to, in order to avoid confusion with a declarative interpretation; wh-questions, on the other hand, are realized with a falling intonation (Hedberg et al., 2010). Nevertheless, Eady and Cooper (1986) report that most of their participants produced a rising intonation for they wh-questions - this may have been due to explicit instruction. Our participants were not instructed to use rising intonation for our polar questions, but did so nevertheless, almost always.
} 
You said that Dillon would help. But now it turns out that $\operatorname{Megan}_{A}$ and $\operatorname{Lauren}_{B}$, or $\operatorname{Morgan}_{C}$ would help?

For the manipulation of focus, we varied the location of a contrast in the two clauses that the speakers produced. For example, in (10) and (11), the second part of the utterance contrasts with respect to the choice of the second name (focus on $B$, or 'second focus'), while in (12) the entire coordinate structure in the second clause contrasts with Dillon in the first clause, so the entire coordinate structure is focused ('broad focus'). Many papers on prosodic focus marking distinguish different types of focus (e.g. Gussenhoven, 2007). Our declarative examples can best be characterized as 'corrective focus' (cf. Ladd, 2008; Vander Klok et al. 2018). Our interrogative examples are similar, in that they involved echo questions, which are closely related to corrective focus in declaratives.

Overall, the experiment involved two different types of speech acts (question vs. declarative), four focus conditions (focus on conjunct A, B, or C, or broad), and two different constituent structures for the coordination $(\mathrm{A}[\mathrm{BC}]$ vs. $[\mathrm{AB}] \mathrm{C})$, for a total of 16 conditions. We had four different item sets varying in these 16 conditions, that differed in lexical content, for a total of 64 different target sentences.

\subsection{Procedure}

The experiment was conducted in a sound-attenuated booth at McGill, using a Logitech H390 headset. Speakers were instructed that the study is about speech production, but were not told that we were specifically interested in prosody and intonation. The experiment was conducted using Matlab scripts developed in our lab for running simple production and perception experiments. On the first screen, 
participants saw instructions about the experiment. They were asked for each trial to first read the target sentences silently, and to proceed with the recording when they felt ready to be recorded. They were instructed to 'say the sentences as naturally as possible, as if you were saying them to a friend in an everyday conversation.' Each utterance consisted of two parts, a set-up sentence and the target sentence. Participants did not get the option of rerecording if they felt unhappy with their first try.

Every participant was recorded on all 64 sentences. The manipulation of speech act was done between two experimental sessions of 32 trials each, which were separated by a filler experiment. About half of the participants were first run on the questions, and the other half were first run on the declaratives. In each session, trials were presented in a pseudo-randomized order maximizing the distance between trials from the same item set, and only allowing maximally one repetition of the same condition.

\subsection{Participants}

A total of 25 native speakers of North American English participated (20 female, five male). They were compensated for their participation. They participated in a language questionnaire about their linguistic background. Eight of the participants reported to be fluent in one or more languages other than English, three reported speaking another language natively. We did not consider linguistic background in analyzing our data, but whether speakers were fluent in another language did not seem to have any obvious differences in the results. 


\subsection{Annotation and Quality Control}

The recorded sentences were manually checked for speech errors, disfluencies, hesitations, and recording errors by three research assistants. Only fluent utterances were kept. This step resulted in the exclusion of $24 \%$ of the data, which were more or less evenly distributed across the various conditions. ${ }^{6}$ The high exclusion rate is likely due to the length and complexity of the stimuli. Due to additional trials where the recording didn't work (probably because participants pressed the key too early), only $70 \%$ of all trials were used (1,166 utterances out of $26 * 64=1,664$ soundfiles).

In order to check whether our manipulations were successful, three research assistants annotated the data. They were asked to decide whether the intonation was falling or rising, what the prosodic phrasing of the coordinate structure was, and which of the four focus prominence options was likely intended. For each dimension, they could also choose 'unclear' if they were not sure. One annotator made a systematic mistake in the annotation of focus and was hence excluded. Inter-annotator agreement between the other two was 'almost perfect' for the annotation of intonation (Cohen's kappa: 0.96), and 'substantial' for constituency (Cohen's kappa: 0.73) and prominence (Cohen's kappa: 0.63).

Overall, the annotation matched very well with the prosodic realization that was expected given the manipulation for all dimensions. One annotator marked the intonational tune according to expectation (that is, declaratives were annotated as falling, and questions as rising) $96.3 \%$ of the time (chance would be 50\%); the expected bracketing $61 \%$ of the time, with about one third of soundfiles marked

\footnotetext{
${ }^{6}$ We included a table with the number of observations per conditions in the supplementary materials.
} 
as 'unclear' (chance would be 50\%); and the expected focus prominence $38 \%$ of the time (chance would be 25\%). The second annotation showed a slightly lower correlation with the original manipulation, but showed a similar overall pattern. These results suggest that our manipulation successfully lead to different prosodic realizations for all three dimensions. The accuracy of the focus prominence annotation is not very high, however. There was a high rate of confusion of broad vs. third focus (that is, focus on the C), and $21 \%$ of utterances marked as 'unclear.' We will discuss this in more detail in section 4 . Since we did not want to bias the results based on prior expectations, we included all of our data in our overall analysis irrespective of whether our annotators were able to map them correctly to the original data, resulting in a total of 1,166 sound files that were included in the analysis.

\subsection{Acoustic Processing of data}

The recordings were aligned with the Montreal Forced Aligner (McAuliffe et al. 2017a), using acoustic models trained on LibriSpeech (Panayotov et al. 2015). The aligned dataset was analyzed with PolyglotDB (McAuliffe et al. 2017b), a software library that can be used for further speech processing. We automatically extracted pitch measures through Praat's $\mathrm{F}_{0}$ analysis, using $50 \mathrm{~Hz}$ and $500 \mathrm{~Hz}$ as the minimum and maxium $\mathrm{F}_{0}$, respectively. Once automatic measures were extracted, each utterance $F_{0}$ track was hand-checked for octave doubling/halving errors with a software interface developed at McGill.7 Intensity

\footnotetext{
${ }^{7}$ To reduce the considerable differences in $\mathrm{F}_{0}$ between speakers and to control for any vowelintrinsic effects on $\mathrm{F}_{0}$, we considered using a relativized measure of mean $\mathrm{F}_{0}$, calculated in PolyglotDB. This relativized measure was a z-score of the $\mathrm{F}_{0}$ using per-speaker means, per-segment means, and standard deviations. The summary statistics were calculated based on all segments in the corpus, including those in words outside the target words (e.g., the relativized measures would
} 
was likewise extracted through PolyglotDB, and relied again on Praat's analysis. Duration was calculated with the forced-aligned boundaries. We decided to use acoustic measures for the first and last syllable of the three target words in the coordinate structure. For each syllable, we extracted the duration, the maximum $\mathrm{F}_{0}$, and the mean intensity.

To illustrate what the recorded utterances were like, let's consider some examples from one of the speakers. Fig. 3 shows two utterances with first focus and a falling declarative intonation, for both types of branching; Fig. 4 shows two utterances with first focus and rising interrogative intonation, for both types of branching.

The utterances clearly convey the post-focal phrasing for both intonational tunes, but they also clearly encode initial focus.$^{8}$ This is visually obvious in the different durations. It is less obvious whether the pitch track carries any independent information about phrasing. In the two utterances with rising question intonation, the focused constituent appears to carry a low pitch accent. The rise of the question involves a final $\mathrm{H} \%$ boundary tone. Note, however, that instead of a gradual rise toward the end of the utterance, there is a sharp rise immediately after the pitch-accented syllable. Similarly, in the utterances with declarative intonation, there is a sharp fall right after the focused constituent. This kind of pattern is analyzed in Pierrehumbert (1980) as being due to a 'phrase accent', which is realized right after the pitch accent, although it is analyzed as a boundary tone per-

adjust for the intrinsic pitch, duration, and intensity of segments, and also for by-speaker variation due to speech rate, gender, etc.). This normalization, however, did not substantially improve the visibility of the effects of interest. In the interest of having easily interpretable measures, we decided to stick to unrelativized measures in this paper. In mixed-effects models, the random effect structure can capture these sources of variability to a large extent anyway.

${ }^{8}$ The example soundfiles are available here: http://prosodylab.org/chael/sounds/jphonExamples/ 


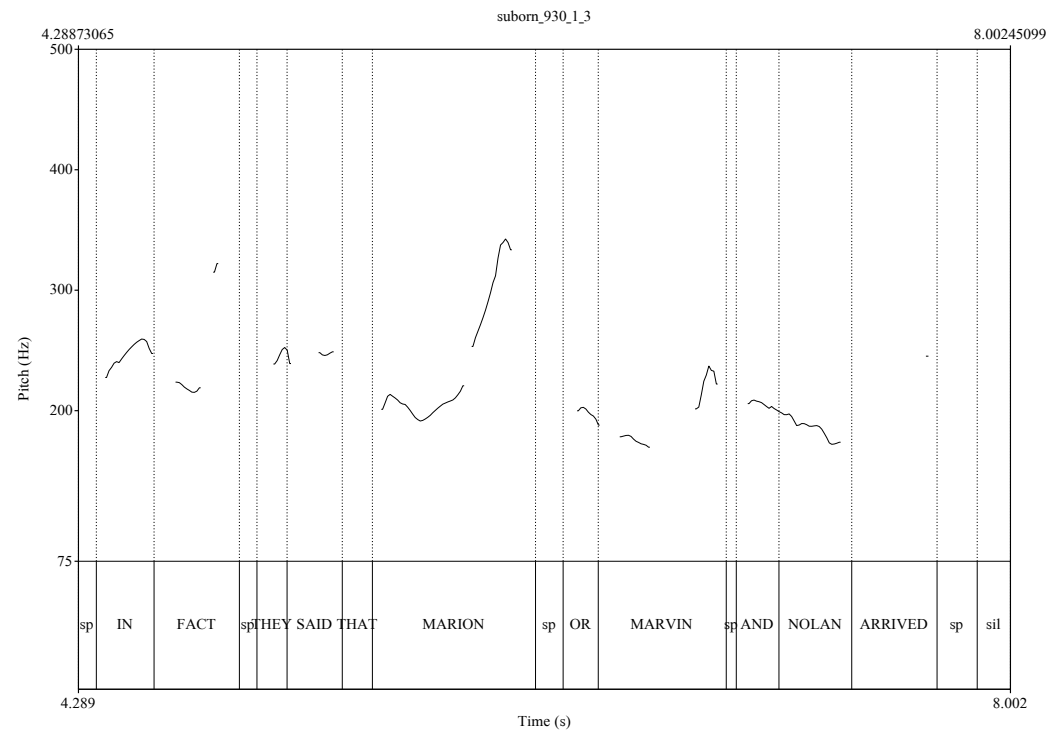

... (Marion or Marvin) and Nolan arrived.

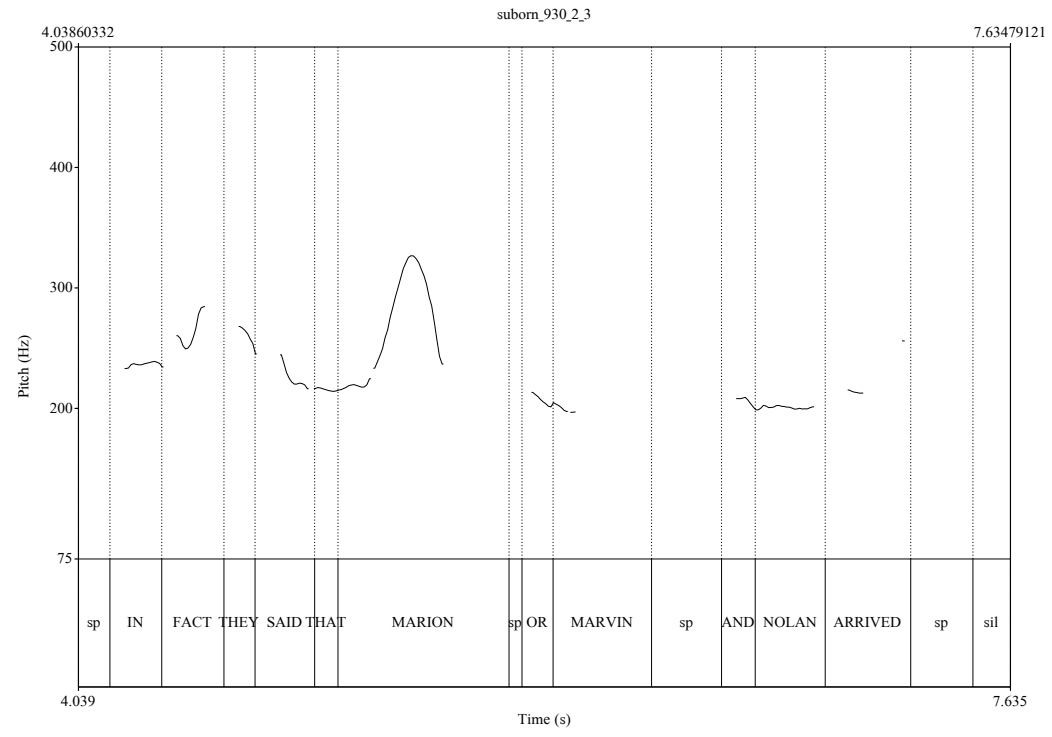

... Marion or (Marvin and Nolan) arrived.

Figure 3: Two examples with initial focus and falling declarative intonation, differing in constituent structure. 


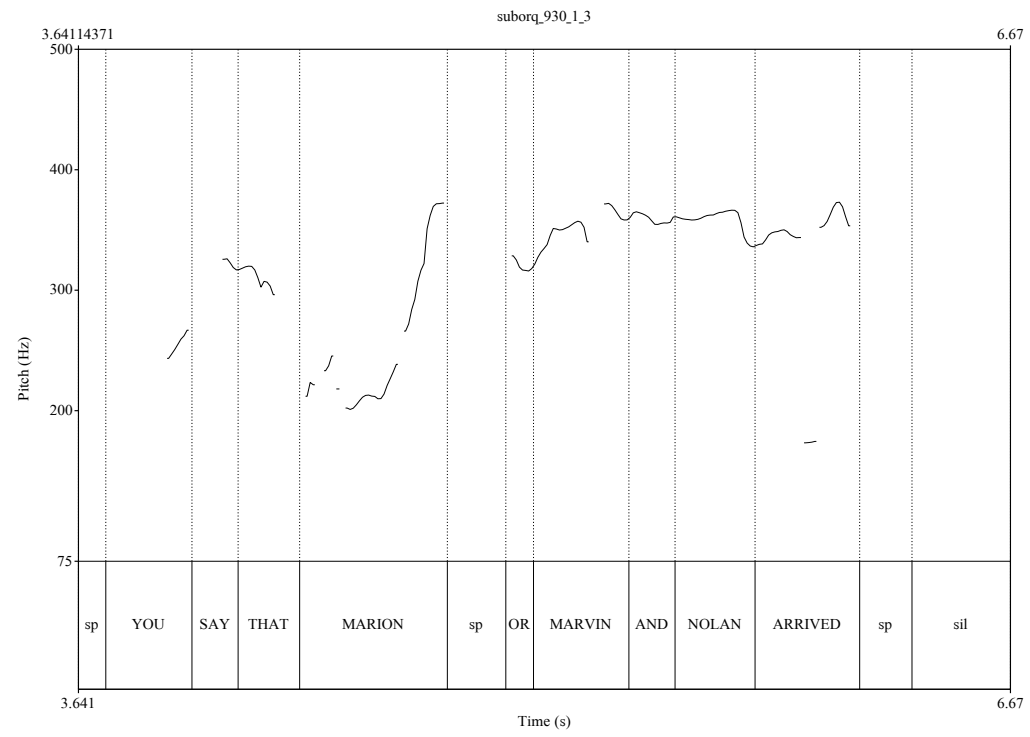

...(Marion or Marvin) and Nolan arrived?

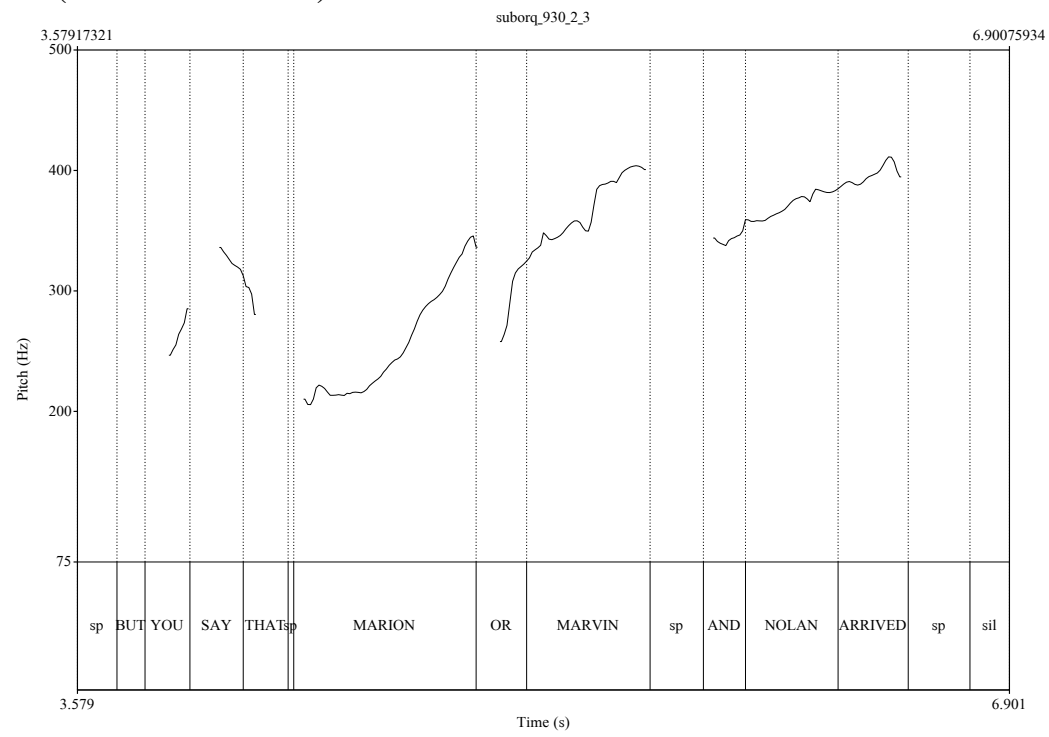

...Marion or (Marvin and Nolan) arrived?

Figure 4: Two examples with initial focus and rising question intonation, differing in constituent structure. 
taining to an intermediate phrase edge. See (Grice et al., 2000) for more evidence for this analysis. Just based on observable tonal events, it seems then that there are no intermediate or intonational phrase boundaries in the post-focal domain, and yet there are clear clues to post-focal phrasing.

\subsection{Results: Durational Effects}

Fig. 5 plots the word durations for our three words of interests, that is, the three names in the coordinate structure. The plot on the left shows the duration of the initial syllable, the one on the right the duration of the final syllable.

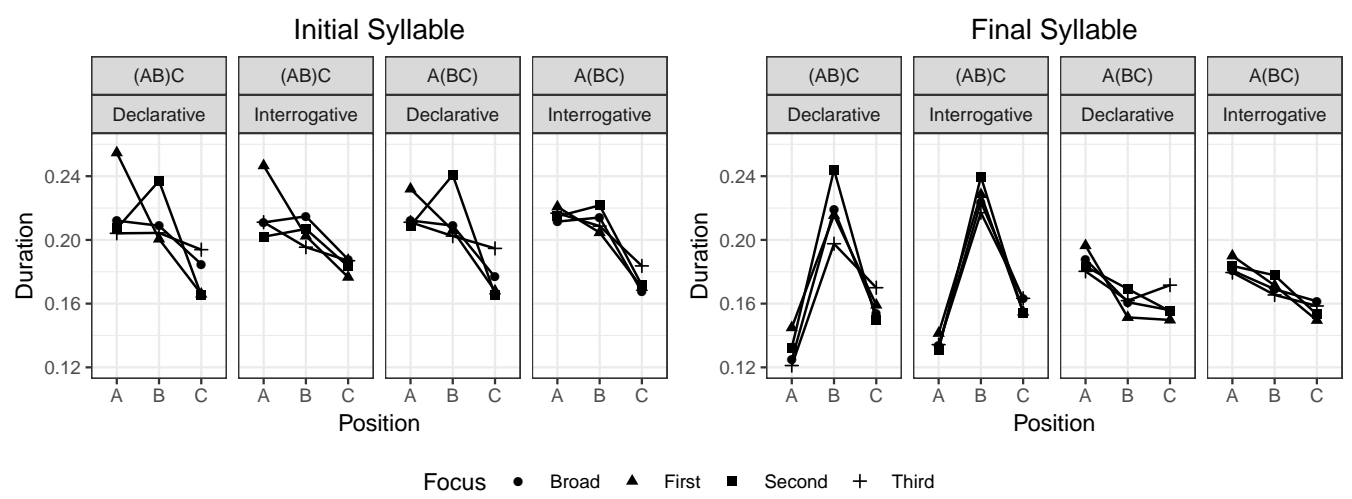

Figure 5: Duration (sec) of the initial and final syllable for each target word

When looking at the durational patterns in the final syllable in Fig. 5, the first pattern that catches the eye is that it closely tracks constituent structure. The plots of all of the combinations of focus and intonation are remarkably uniform for both left-branching and right-branching coordinate structures. If $A$ forms a constituent with $B$, then the final syllable of $\mathrm{A}$ is much shorter and that of $\mathrm{B}$ much longer compared to the case in which $B$ forms a constituent with $C$. This makes sense 
if constituent structure maps to prosodic phrasing such that immediate conjuncts are phrased together to the exclusion of other constituents, and if the amount of lengthening on the final syllable correlates with whether the following constituent is in a separate phrase or in the same phrase. Notably, the durational effects of phrasing do not appear to be diminished by initial focus. This suggests, as was found already in Norcliffe and Jaeger (2005), that durational cues to phrasing remain intact in the post-focal domain.

The plot of the durational effects on the initial syllable do not show any obvious correlates of phrasing or intonation, but they do show that duration provides cues for focus. At least in declarative utterances, the initial syllable of the focused word shows a boost in duration for all three focus conditions, compared to the baseline with broad focus. In the interrogative cases, this is only apparent when the initial constituent is focused (so for constituent A in the initial focus condition). The boost in duration of the focused initial syllable is much smaller on the final word C. Cooper et al.(1985) also observed that final words show less focusrelated lengthening compared to non-final words. Syllables other than the initial syllable of the focused word seem to be mostly unaffected by focus, and post-focal words for the most part do not show a reduction in duration. This again fits with Cooper et al. (1985), who similarly found that the effect of focus on duration is largely confined to the accented syllable of the focused word 9

The plots suggest an interaction of focus with intonation: The different foci seem less differentiated by duration with interrogative intonation compared to declarative intonation.

\footnotetext{
${ }^{9}$ In our data, a small reduction in duration when post-focal is only observed for word C (but not for word B).
} 
In order to further evaluate the data, we fitted a regression model of the duration of initial and final syllables of word B, summarized in Table $1^{10}$ The models we fit had duration (in seconds) as the dependent variable, and speech act, constituency, intonation, and their interactions as fixed effects. The model also included random slopes for all fixed effects, both for items and participants. We estimated p-values using the Satterthwaite approximation to estimate the degrees of freedom (Kuznetsova et al., 2013) (p-values were rounded to 0.001 or higher).

\begin{tabular}{lcc}
\hline & Initial & Final \\
\hline (Intercept) & $0.21(0.04)^{* *}$ & $0.19(0.02)^{* *}$ \\
Broad.vs.Narrow & $0.00(0.00)$ & $-0.00(0.00)$ \\
First.vs.Late & $0.01(0.01)$ & $0.00(0.00)$ \\
Second.vs.Third & $-0.02(0.01)^{* * *}$ & $-0.02(0.01)^{* * *}$ \\
Decl.vs.Inter & $-0.00(0.00)$ & $0.01(0.00)^{* *}$ \\
Left.vs.Right & $0.00(0.00)$ & $-0.06(0.01)^{* * *}$ \\
Broad.vs.Narrow:Decl.vs.Inter & $-0.01(0.00)$ & $-0.00(0.01)$ \\
First.vs.Late:Decl.vs.Inter & $-0.01(0.01)$ & $-0.01(0.01)^{*}$ \\
Second.vs.Third:Decl.vs.Inter & $0.03(0.01)^{* * *}$ & $0.01(0.01)$ \\
Broad.vs.Narrow:Left.vs.Right & $0.01(0.00)$ & $-0.00(0.01)$ \\
First.vs.Late:Left.vs.Right & $-0.00(0.01)$ & $0.01(0.01)$ \\
Second.vs.Third:Left.vs.Right & $0.00(0.01)$ & $0.02(0.01)^{* *}$ \\
Decl.vs.Inter:Left.vs.Right & $0.00(0.00)$ & $0.00(0.00)$ \\
Broad.vs.Narrow:Decl.vs.Inter:Left.vs.Right & $-0.00(0.01)$ & $0.00(0.01)$ \\
First.vs.Late:Decl.vs.Inter:Left.vs.Right & $0.01(0.01)$ & $-0.01(0.01)$ \\
Second.vs.Third:Decl.vs.Inter:Left.vs.Right & $0.00(0.01)$ & $-0.03(0.01)^{*}$ \\
\hline${ }^{* * * *} p<0.001,{ }^{* * *} p<0.01$, $^{*} p<0.05$ & &
\end{tabular}

Table 1: Mixed Effects Regression Models for the duration of word B (estimates in sec, SE in parentheses)

The results for the initial syllable of word B show an effect of focus, such that

\footnotetext{
${ }^{10} \mathrm{We}$ focus here on measures of word B because based on the empirical plots, it seems like this is the most informative word. Looking at each word of interest would have made this paper too long. But of course analysis of the other words would also be of interest. We report additional models in the supplementary materials. We used the package lme4 (version 1.1-18-1) to fit the models, and estimated p-values with the package lmerTest (Kuznetsova et al. 2013).
} 
with focus on B (second focus), the initial syllable was about $23 \mathrm{~ms}$ longer than with focus on $\mathrm{C}(\beta=-0.023 ; S E=0.005 ; t=-3.8 ; p<0.001)$.11 There was also a significant interaction with intonation $(\beta=0.026 ; S E=0.006 ; t=4.5 ; p<$ 0.001), indicating that this lengthening of the initial syllable due to focus was significantly smaller in questions.

The results for the final syllable of word $\mathrm{B}$ also show a main effect of focus, similar in magnitude to that in the initial syllable $(\beta=-0.022 ; S E=$ $0.005 ;-t=-4.3 ; p<0.001)$. There was a main effect of intonation, such that the final syllable was significantly longer in questions $(\beta=0.096 ; S E=$ $0.003 ; t=-3.1 ; p<0.005)$, maybe because word $\mathrm{B}$ was typically realized with a rising pitch accent, which may require a longer final syllable. There was also a sizeable effect of phrasing, such that in the left-branching structure, the final syllable was significantly longer than in the right-branching structure $(\beta=-0.055 ; S E=0.008 ; t=-6.5 ; p<0.001)$. In other words, the syllable was on average about 55ms longer when B was phrase-final and formed a constituent with A. There was also a small but significant interaction effect between first vs. late focus and intonation $(\beta=-0.012 ; S E=0.006 ; t=-1.97 ; p<0.05)$, indicating that the choice of tune indeed significantly affected the realization of focus, with a smaller effect of focus in polar questions 12

If placing focus on A would erase the prosodic phrasing in the remainder of the utterance, then B should not vary depending on phrasing with first focus. An inter-

\footnotetext{
${ }^{11}$ Eady and Cooper (1986) found that the accented syllable of a word was lengthened by about $30 \mathrm{~ms}$ under focus.

${ }^{12}$ There were two further interaction effects, for which we do not have an interpretation: There was an interaction between the realization of Second.vs.Third focus and branchingness; and a three-way interaction between second vs. third focus, intonation, and syntactic constituent structure.
} 
action between the effect of First.vs.Late Focus and branchingness (Left.vs.Right) could have provided evidence for such a post-focal dephrasing effect. We did not, however, find evidence for such an effect.

\subsection{Results: Intensity Effects}

Turning to intensity, Fig. 6 illustrates that the intensity of the final syllable provides a consistent cue to prosodic phrasing, across all conditions. The observed effects of phrasing on intensity are a mirror-image of the duration effects: The final syllable of word A shows higher intensity than that of word B when they phrase together as a constituent, and lower intensity when they do not. This pattern cannot be explained by a simple gradual drop in intensity within phrases: The first constituent A, for example, should in that case always be realized in the same way across different phrasings. Instead, it seems that relative intensity is actively scaled to encode the prosodic phrasing of the utterance.

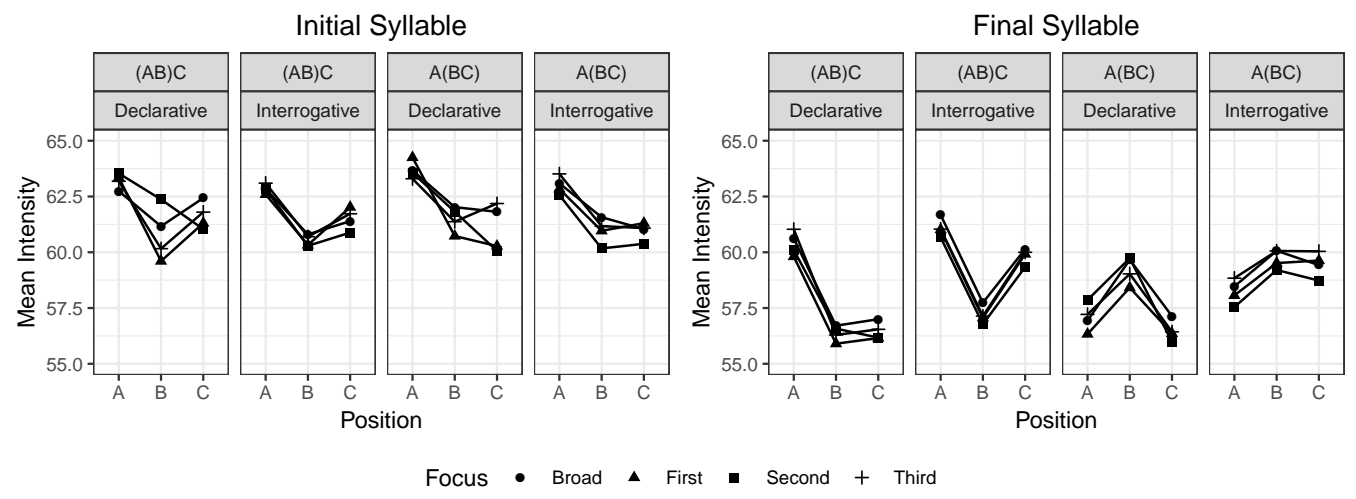

Figure 6: Mean intensity $(\mathrm{dB})$ of initial and final syllable for each target word.

A second clear pattern in the plot is that the intensity of the final syllable of word $\mathrm{C}$ is higher in the interrogative condition compared to the declarative condi- 
tion. This intensity increase may be an indirect consequence of the increased $\mathrm{F}_{0}$ for the final rise. Increasing the rate of glottal fold vibration requires an increase in sub-glottal pressure, and may hence also result in greater intensity. Although intensity is typically not thought of as a cue to distinguish declarative and interrogative intonation, it seems clear that it is at least a reliable secondary cue in our data.

The intensity pattern on the initial syllable is less clear. It seems that focus affects its intensity at least in the declarative condition, but the pattern is quite different depending on phrasing. The intensity of the initial syllable does not appear to vary by intonation.

We again fitted models for word B to further evaluate the effects, which are reported in Table 2 .

\begin{tabular}{lcc}
\hline & Initial & Final \\
\hline (Intercept) & $60.47(1.36)^{* * *}$ & $57.69(1.24)^{* * *}$ \\
Broad.vs.Narrow & $-0.61(0.16)^{* *}$ & $-0.53(0.21)$ \\
First.vs.Late & $0.75(0.24)^{*}$ & $0.45(0.29)$ \\
Second.vs.Third & $-0.38(0.30)$ & $-0.15(0.57)$ \\
Decl.vs.Inter & $-0.24(0.29)$ & $0.79(0.43)$ \\
Left.vs.Right & $0.73(0.21)^{* *}$ & $2.73(0.60)^{* * *}$ \\
Broad.vs.Narrow:Decl.vs.Inter & $0.20(0.32)^{* *}$ & $0.27(0.33)$ \\
First.vs.Late:Decl.vs.Inter & $-1.23(0.35)^{* * *}$ & $-0.57(0.37)$ \\
Second.vs.Third:Decl.vs.Inter & $0.87(0.40)^{*}$ & $0.20(0.42)$ \\
Broad.vs.Narrow:Left.vs.Right & $-0.42(0.32)$ & $-0.04(0.33)$ \\
First.vs.Late:Left.vs.Right & $-0.68(0.35)$ & $0.14(0.37)$ \\
Second.vs.Third:Left.vs.Right & $0.52(0.40)$ & $-0.30(0.41)$ \\
Decl.vs.Inter:Left.vs.Right & $0.05(0.28)$ & $-0.19(0.30)$ \\
Broad.vs.Narrow:Decl.vs.Inter:Left.vs.Right & $-0.72(0.64)$ & $-0.47(0.67)$ \\
First.vs.Late:Decl.vs.Inter:Left.vs.Right & $0.35(0.71)$ & $-0.31(0.73)$ \\
Second.vs.Third:Decl.vs.Inter:Left.vs.Right & $-1.17(0.80)$ & $0.60(0.83)$ \\
\hline$* * * *<001 * * * 001$ * $p<0.05$ & &
\end{tabular}

Table 2: Mixed Effects Regression Models for the mean intensity of word B (estimate in dB, SE in parentheses). 
For the initial syllable, there was a significant effect of First.vs.Late focus, such that the initial syllable of word B had lower intensity if the first conjunct was focused $(\beta=0.75 ; S E=0.24 ; t=3.1 ; p<0.05)$ showing that post-focal material is reduced 13

There was an effect of similar size of phrasing $(\beta=0.73 ; S E=0.21 ; t=$ 3.5; $p<0.02$ ), indicating that the intensity of the initial syllable of word B was 'scaled' to encode the constituent structure: B had lower intensity when it was phrase-final (in the left-branching structure), compared to when it was phraseinitial (in the right-branching structure). There were furthermore two interaction effects between how focus was realized and the choice of intonation, suggesting that intensity was a significantly less reliable cue for focus in interrogative utterances.

For the final syllable, there was only one significant effect. The final syllable of word B, just like its initial syllable, showed a lower intensity in the left-branching structure, where it was phrase-final, compared to the right-branching structure, where it was phrase-intial $(\beta=2.73 ; S E=0.6 ; t=4.5 ; p<0.001)$.

If focus on the first constituent were to obliterate phrasing later, then we would expect an interaction between First.vs.Late and Left-vs.Right. Just as in the case of duration, we failed to find evidence for such an interaction.

\footnotetext{
${ }^{13}$ There was also a significant effect of Broad.vs.Narrow, such that the initial syllable of word $\mathrm{B}$ was longer under narrow focus compared to the control condition $(\beta=-0.61 ; S E=0.16 ; t=$ $-3.8 ; p<0.002)$. We would have expected that the comparison between Second.vs.Third would be significant instead.
} 


\subsection{Results: $F_{0}$ Effects}

In declaratives, focus clearly affects the $\mathrm{F}_{0}$-pattern of the initial syllable of the target words. The initial syllable of a focused word is generally boosted compared to the broad focus baseline for all three target words A, B, and C. This replicates similar effects reported in the literature (Eady and Cooper, 1986; Breen et al., 2010, i.a.) ${ }^{14}$ There was one exception, however: With third focus, in the left-branching structure, the pitch on the initial syllable of the focused word $\mathrm{C}$ is similar to the baseline 15

The pitch of the initial syllable appears to be systematically lowered when a word is post-focal, such that the initial syllable of B is lower compared to baseline when $\mathrm{A}$ is focused, and the initial syllable of $\mathrm{C}$ is lowered when $\mathrm{A}$ or $\mathrm{B}$ are focused. By contrast, there is only little evidence for pre-focal reduction: Pitch on the initial syllable of word A is comparable to baseline when focus is placed on $\mathrm{B}$ and $\mathrm{C}$; pitch on the initial syllable of word $\mathrm{B}$ is only reduced for third focus in the left-branching structure, but is identical to baseline in the right-branching structure. Earlier studies also found that pitch reduction is stronger post-focally compared to pre-focally Eady and Cooper (1986); Eady et al. (1986), an observation to which we return when looking at human annotations of the data in the next section.

\footnotetext{
${ }^{14}$ Interestingly, Eady and Cooper (1986) found no boost in pitch for initial constituents. A relevant difference to our study is, however, that our target sentence was not the only clause the participants produced. Word A was clause-initial, but not utterance-initial, while in their study the initial word of interest they looked at was utterance-initial.

${ }^{15}$ Maybe relatedly, Eady and Cooper (1986) report that pitch was boosted on the final word with late focus, while Cooper et al. (1985) report that this is not the case in longer utterances, where the maximum pitch is similar to baseline in this case. It's not clear why our left-branching (as opposed to right-branching) cases should pattern with the longer sentences in their studies, but it is interesting that a similar point of variation was observed before.
} 


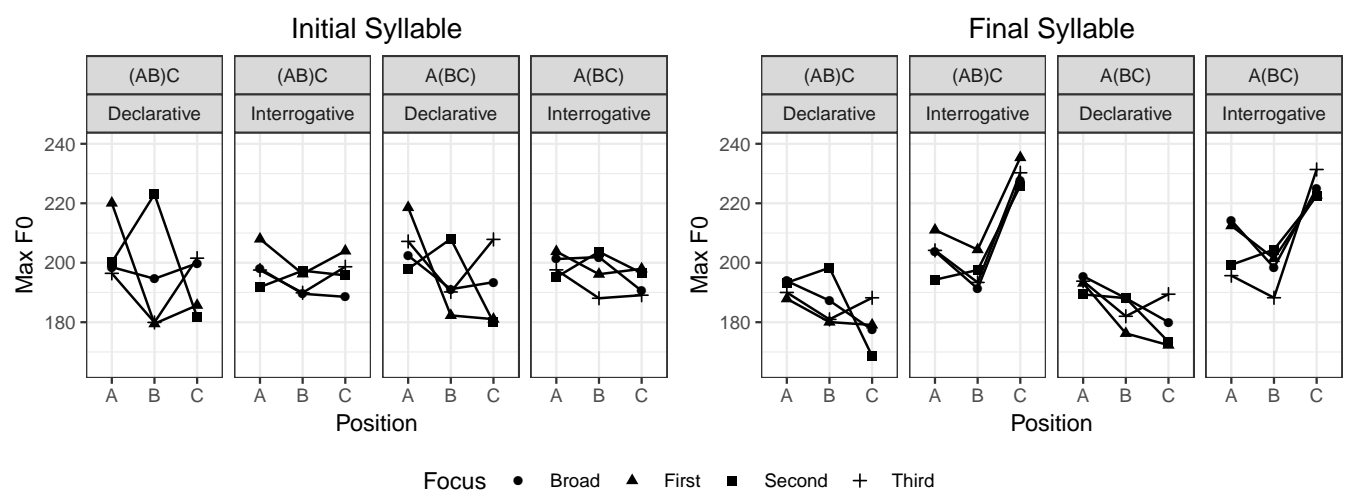

Figure 7: $\mathrm{Max}_{0}(\mathrm{~Hz})$ of initial and final syllable for each target word

Systematic effects of phrasing and intonation are not apparent for the initial syllable. The pattern of $F_{0}$ on the final syllable, however, shows clear effects of the intonational contour: The pitch of the final syllable of word C is substantially higher in interrogatives, reflecting the final high boundary tone of the question contour. $\mathrm{F}_{0}$ on the final syllable of word $\mathrm{A}$ and $\mathrm{B}$ appears to be higher in interrogatives as well, compatible with these words carrying rising accents. The effects of focus and phrasing on the final syllable seem comparatively weak, if present at all.

We again fitted a regression model for word $\mathrm{B}$, reported in Table 3 . The model for the initial syllable shows a significant effect of First.vs.Late focus, confirming that the initial syllable of word B was indeed significantly reduced (by about $10 \mathrm{~Hz})$ when the first word was focused $(\beta=9.6 ; S E=3.2 ; t=-3.0 ; p<0.007)$. There was also a significant effect of Second.vs. Third focus, confirming that pitch of the initial syllable of word B was significantly boosted when it was focused $(\beta=-19.4 ; S E=4.8 ; t=-4.0 ; p<0.001)$. There were also highly signifi- 
cant interactions of focus with intonation (First.vs.Second:Decl.vs.Inter and Second.vs.Third:Decl.vs.Inter), showing that focus was cued less reliably in questions 16

\begin{tabular}{lcc}
\hline & Initial & Final \\
\hline (Intercept) & $192.50(7.77)^{* * *}$ & $189.56(8.45)^{* * *}$ \\
Broad.vs.Narrow & $0.88(3.11)$ & $-0.13(1.70)$ \\
First.vs.Late & $9.63(3.25)^{* *}$ & $1.93(4.26)$ \\
Second.vs.Third & $-19.41(4.81)^{* * *}$ & $-11.01(4.69)$ \\
Decl.vs.Inter & $1.46(3.27)$ & $12.23(4.51)^{*}$ \\
Left.vs.Right & $2.40(2.06)$ & $0.16(2.19)$ \\
Broad.vs.Narrow:Decl.vs.Inter & $1.05(3.22)$ & $9.88(3.41)^{* *}$ \\
First.vs.Late:Decl.vs.Inter & $-18.61(3.53)^{* * *}$ & $-12.58(3.78)^{* * *}$ \\
Second.vs.Third:Decl.vs.Inter & $18.88(4.04)^{* * *}$ & $0.58(4.29)$ \\
Broad.vs.Narrow:Left.vs.Right & $-1.98(3.21)$ & $-4.03(3.41)$ \\
First.vs.Late:Left.vs.Right & $-3.67(3.53)$ & $-0.24(3.78)$ \\
Second.vs.Third:Left.vs.Right & $5.87(4.01)$ & $-1.47(4.26)$ \\
Decl.vs.Inter:Left.vs.Right & $5.96(2.85)^{*}$ & $3.35(3.04)$ \\
Broad.vs.Narrow:Decl.vs.Inter:Left.vs.Right & $-9.62(6.42)$ & $1.86(6.82)$ \\
First.vs.Late:Decl.vs.Inter:Left.vs.Right & $7.19(7.06)$ & $0.95(7.57)$ \\
Second.vs.Third:Decl.vs.Inter:Left.vs.Right & $-19.07(8.02)^{*}$ & $-9.16(8.54)$ \\
\hline
\end{tabular}

Table 3: Mixed Effects Regression Models for the Max $\mathrm{F}_{0}$ of word $\mathrm{B}$ (estimate in $\mathrm{Hz}, \mathrm{SE}$ in parentheses).

For the final syllable, there was an effect of intonation $(\beta=-12.59 ; S E=$ $3.9 ; t=3.2 ; p<0.01)$. There were also interactions between First.vs.Late Focus and Second.vs.Third Focus. Notably, there were no significant effects of phrasing on pitch in either syllable.

\subsection{Discussion}

Our results show that duration, intensity, and pitch are all affected by each of the three functional dimensions, speech act, constituent structure, and focus.

\footnotetext{
${ }^{16}$ There was also a significant interaction between intonation and branching, as well as a significant three-way interaction, for which we do not have an interpretation.
} 
There are two patterns that make it easier to tease apart the three dimensions, despite the overlap in which acoustic cues they affect. The first is that the three dimensions tend to affect different syllables. Focus prominence mostly affects the accented syllable, constituent structure mostly affects the final syllable, and intonation affects both. The second pattern is that the the acoustic cues have different relationships with each other depending on the dimension. For example, duration, pitch and intensity of the initial syllable all increase when a word is focused; however, when a word is phrase-final, duration increases while intensity decreases (and we did not find a pitch effect). In other words, the cues mutually inform each other about which dimension is being cued. A listener can draw different conclusions depending on whether increase in duration is accompanied by an increase in intensity or a decrease .17

Our main research question was whether focus prominence obliterates cues to phrasing in the post-focal domain. Our data show no evidence for this. Based on the empirical plots, the duration of the final syllable of our target words consistently reflect phrasing, unperturbed by variation in focus or intonation. The intensity of both the (accented) initial syllable and the final syllable also varies by phrasing. We found main effects of phrasing for several of our measures, but for no acoustic measure did we find an interaction between the effect of constituency and the effect of focus. There is no evidence therefore that focus erases or diminishes phrasing distinctions in the post-focal domain.

In addition, we found that focus is cued less reliably with interrogative intona-

\footnotetext{
${ }^{17}$ This difference in relation with each other may be at the heart of the interaction-effect observed between duration and intensity in the logistic regression fitted to predict prominence judgments in Bishop et al. (this special issue).
} 
tion. It seems then that question intonation tends to diminish cues to focus at least to some extent. We explore these two findings in more detail the next section. Our results differ in this regard from findings in Eady and Cooper (1986), where the choice between declarative intonation and question intonation did not affect the durational cues to focus, and no interaction with intonation was observed. An important difference in their study, however, is that when the experimenter thought that focus was not successfully conveyed prosodically, speakers were instructed to rerecord a sentence. In the next section, we use the effect of intonation on focus in our data as a point of comparison to evaluate whether there might be an effect of focus on phrasing that remained undetected by our models. Before doing so, we want to take stock of a few findings that are not our main focus, but are nevertheless of interest.

An interesting parallel in our results to those of Cooper et al. (1985) and Eady and Cooper (1986) is that while durational cues to focus are confined (largely) to the accented syllable of the focused word, the pitch cues to focus are more globally distributed, since post-focal material is also affected. Our results furthermore show that in this regard intensity patterns more similarly to pitch, in that in addition to the boost in intensity on focused material, post-focal material is also reduced in intensity.

Furthermore, we observed an interesting pattern regarding the phrasing-related lengthening effect that affects the last syllable of a word. We found a much greater effect of final lengthening in pre-boundary environments, when another conjunct followed, compared to the final conjunct. This replicates similar findings in Wagner (2005), and suggests that these are planning-related pre-boundary effects rather than (just) effects of final lengthening. Note, however, that in our ut- 
terances there was material following the final conjunct, which was contextually given and hence unaccented, which may explain why the observed final lengthening in word $\mathrm{C}$ was not greater 18

This result relates to the comparatively small degree of lengthening for focus reasons we found in the final word: Cooper et al. (1985), similarly, found that final words show less of a durational increase under focus, and interpret this as a ceiling effect-the final word is subject to final lengthening since it is phrasefinal, and cannot expand much in addition to also encode focus. This explanation does not seem applicable in our case: Since we didn't find evidence for phrasingrelated lengthening in the final word, there is no reason to assume that a ceiling in duration was reached. Also, it is not clear why a ceiling should be reached for lengthening the initial syllable anyway, when phrase-related lengthening mostly affects the final syllable. This casts doubt on an explanation based on overall expandability. An alternative interpretation of the smaller focus effect on the last word is that the last accented word is perceived as sufficiently prominent for a focused word simply by virtue of being last, making it less important to mark late focus phonetically.

Another interesting finding is that phrasing seems to be reliably cued by intensity-scaling. Both the initial accented syllable and the final unaccented syllable show clear effects of phrasing, such that there is higher intensity at the beginning of phrases and lower intensity later in phrases. There is clear evidence that

\footnotetext{
${ }^{18} \mathrm{~A}$ reviewer points out that the reason for the increased effect coordinate-structure internally could be that speakers are trying to disambiguate the globally ambiguous structure. Our data do not allow to test whether this is the case. Another reviewer suggests that there may be greater lengthening phrase-internally because there may be no clear tonal cue signalling the phrase boundary while intermediate-phrase finally, there is a boundary tone. See Gollrad (2013) for evidence that pitch only plays a minor role in cueing intermediate phrase boundaries in German.
} 
these effects go beyond a drop in sub-glottal pressure due to exhalation throughout the utterance or throughout phrases. We would not expect to see a difference in the intensity on the first constituent (A) if this were the case. And yet, Fig. 6 illustrates that A varies in intensity depending on whether it phrases with B or not. It seems then that intensity-scaling is actively used to encode prosodic phrasing. This is compatible with related findings in German reported in Poschmann and Wagner (2016).

The lack of pitch accent scaling effects due to phrasing, and the clear presence of intensity-scaling, is compatible with the hypothesis that intensity is a primary cue for phrasing, while apparent effects of pitch-accent scaling observed in the earlier literature may really have been an indirect side-effect of intensity scaling. Intensity in speech closely correlates with pitch (Gramming et al., 1988). The correlation with pitch is not a physical necessity, of course, and individuals seem to have independent control over the two cues-we are able in principle to talk louder or quieter without changing $\mathrm{F}_{0}$. And yet, when asked to speak louder, speakers often spontaneously raise their pitch. It seems that an increased sub-glottal pressure will result in higher vocal fold vibration unless we prevent this from happening. The close correlation between the two cues opens the possibility that pitch may be used to enhance an intended intensity effect and vice-versa. Maybe pitch accent scaling is only an indirect correlate of intensity scaling. Further investigation of this idea is needed. Our results minimally suggest that intensity-scaling is a stronger and more reliable cue to phrasing than pitch-scaling. In our data, pitch scaling appears not to be a good to cue to phrasing, even in the case of broad focus 19

\footnotetext{
${ }^{19}$ Note that other pitch measures could be explored, including rate of pitch change or range, see
} 
Pitch cues to differences in phonological phrasing were also not found to be very relevant in the perception studies conducted in Gollrad (2013) on German. However, Gollrad (2013) found that pitch cues related to boundary tones were much more relevant to cue intonational phrasing than in phonological phrasing. It seems plausible that in our data, intonational phrase breaks were used less frequently than phonological phrase boundaries to mark the intended phrasing, given the shortness of the conjuncts. Maybe we would have found reliable pitch cues if we had looked at phrasing distinctions among larger constituents that map to intonational phrases, as Ladd (1988) did in his classic study on pitch scaling.

Overlay models predict the prosodic reflexes of the three separate dimensions to be independent and recoverable from the signal, while most current phonological models predict interactions resulting in neutralizations between conditions. We found no evidence that phrasing distinctions are neutralized in the post-focal domain. The plots suggest that focus and phrasing are, for the most part, encoded independently of each other, as expected by overlay models. It should be noted that such additive effects of different factors are not inherently incompatible with AM-models. Pierrehumbert (1980, 18), for example, anticipates that the expressive use of pitch of a speaker trying to 'highlight' certain parts of the utterance might 'multiplicatively determine the $F_{0}$ value of a given tone'. We will discuss particular proposals about phonological presentations compatible with our findings in section 4.3 .

How compelling is the evidence that the prosodic cues to phrasing and focus are orthogonal to each other? The lack of significant interaction effects is only of limited informativeness. We cannot rule out that our experiment was simply

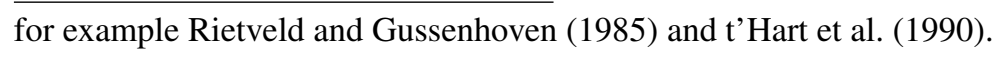


not powerful enough to detect such effects. At least the empirical plots show highly consistent patterns across conditions, and make it seem implausible that focus has radical effects on post-focal phrasing. We can do better, however. In the next section, we will try to quantify just how well various acoustic cues convey phrasing, and also how much loss of information there is when focus is placed early. This will also address a second worry one may have based on the results reported above: By looking only at a few acoustic cues from the second conjunct (word B), we have used a very limited amount of information from the signal.

\section{How well is phrasing cued post-focally?}

In order to evaluate to what extent information about phrasing in the post-focal domain is lost, we need to find a way to look at many acoustic cues at the same time, and evaluate how they fare in cueing phrasing for different focus conditions. One way we can accomplish this is by automatically classifying the data given a set of acoustic features. There are many different types of classifying algorithms. Here, we will use Random Forests (Breiman, 2001; Strobl et al., 2009). Random forests have been used insightfully in earlier work in linguistics (Tagliamonte and Baayen, 2012; Tao et al., 2018; Baumann and Winter, 2018), including in this special issue (Wagner et al., this special issue).

\subsection{Methods}

Random forests provide a non-parametric statistical tool which allows us to evaluate a set of features (a 'feature space') in its usefulness in classifying observations into pre-set categories. Compared to alternative analysis methods, they have the advantage of being robust with respect to collinearity among the predictors, and in being compatible with a large set of predictors with a relatively 
low $n$ (Strobl et al. 2009). The former property is important if one wants to explore the contributions of both pitch and intensity, since they are generally highly correlated, as noted before.

A random forest consists of an 'ensemble' or 'forest' of classification and regression trees (CART, Breiman et al.|1984). The trees in the forest vary in which subset of the data they were trained on, and which subset of predictors were used. CARTs classify the data by recursive splits that try to optimally sort the data into the correct bins. A disadvantage of CARTs is that the ranking of predictors is very sensitive to small changes in the data used to train them. Each split at a given point in the classification tree is very sensitive to the particular training data, and can have large downstream effects on which features will be chosen. If different subsets are used to train a CART, very different classification trees may emerge. In any given tree, a particular predictor may be masked by others, even if, when faced with novel data, that predictor might have been very useful. Similar issues can arise in stepwise predictor-selection algorithms in logistic regression models. Random forests avoid this issue by fitting many CARTs based on different subsets of the data. One can then evaluate the importance of particular predictors over the entire forest. In addition, they increase the variability of trees even further by restricting the set of predictor variables for each individual tree, which helps to estimate their relative usefulness even more accurately.

Random forests have two parameters, the number of trees ('ntree'), and the number of randomly preselected splitting variables for each tree ('mtry'). Small data sets may require a larger number of trees to find robust results. A low number of 'mtry' increases tree diversity. This is because we select a small number of predictors, it is more likely that more important features are missing, which will 
give the 'lesser' features an opportunity to show their worth. However, it leads to more trees in which all relevant predictors are left out, introducing more noise. A high value for this parameter means that the best predictors will usually be chosen, and thus tree diversity decreases, and along with it the quality of information gained on lesser features (for more discussion, see Strobl et al., 2009).

Random forests have two more useful properties. First, they allow us to estimate how well a set of features differentiates different categories by testing how well data is classified that was left out of the training set for each tree during the training phrase. This is called 'out-of-bag' (OOB) prediction. Second, they allow us to evaluate the relative importance of particular predictor variables. This is done by permuting the values of particular predictors and evaluating how this affects the classification accuracy on out-of-bag data. If a predictor is important for the classification, permuting its value will greatly affect out-of-bag classification accuracy. If it was involved in a tree by chance, then it will not. These permutations yield variable importance measures for each predictor. The absolute values of these importance measures are hard to interpret, but the relative ranking is meaningful. It tells us which features were most important in the model. If a variable has an importance close to zero, it does not contribute much to the accuracy of the predictions. If it is negative, then leaving it out of a classification tree improves the classification.

In order to first understand which predictors were important to encode focus, we fitted a random forest to predict the original focus context that an utterance was uttered in. The predictors included duration, pitch, and intensity measures for each of the three target words for each syllable, for a total of 18 measures. Following the heuristic in Strobl et al. (2009), we set the parameter mtry to the rounded 
square root of the number of predictors $(m t r y=4)$. We used forests of 1000 trees. We also ran the analysis with different parameters $(\operatorname{mtry}=3 \& 5$, ntrees $=5,000)$ to ensure that the results are stable 20

In analysis based on mixed models, we have seen that focus seems to have had less of an effect in interrogative utterances. Therefore, we ran the analysis not just on the entire data, but also on the subset of declarative utterances and interrogative utterances respectively, in order to assess how the choice of intonation affected the accuracy of the classification. The goal was to see how much information about focus is still present in the interrogative utterances, compared to the declaratives. We then proceeded to look at our central question, which is how much information about phrasing is lost in the post-focal domain, and fitted a random forest model to predict phrasing. Here, we were interested whether the classification accuracy was lower when focus was initial compared to the case of broad focus-if initial focus adversely affects cues to phrasing, the classification for phrasing should be lower.

\subsection{Results}

Table 4 illustrates how well the random forest performed compared to a human annotator, tasked with guessing which focus condition an utterance was recorded in 21

For the declaratives, the random forest classifier has an accuracy between $31 \%$ and $44 \%$. This is not spectacular, but much better than chance, which is at $25 \%$.

\footnotetext{
${ }^{20} \mathrm{We}$ used the R party-package to compute the random forest analysis (Hothorn et al., 2006, Strobl et al. 2007. 2008). Apart from the cited literature, we also thank Stephanie Shih and Francisco Torreira for their useful class materials on random forest analysis.

${ }^{21} \mathrm{We}$ do not report the accuracy on the training data since these accuracy percentages are inflated, and will not reflect how well the model would do when encountering new data.
} 


\begin{tabular}{lllll}
\hline & Focus & R.F./Annotator-All & R.F./Annotator-Dec & R.F./Annotator-Int \\
\hline 1 & Broad & $0.25 / 0.16$ & $0.31 / 0.14$ & $0.19 / 0.16$ \\
2 & First & $0.38 / 0.35$ & $0.42 / 0.39$ & $0.27 / 0.26$ \\
3 & Second & $0.37 / 0.36$ & $0.45 / 0.45$ & $0.3 / 0.24$ \\
4 & Third & $0.35 / 0.6$ & $0.33 / 0.66$ & $0.22 / 0.49$ \\
\hline
\end{tabular}

Table 4: Proportion of accurate classification for all data, declarative data (Dec), and interrogative data (Int). For each data set, both the accuracy of the random forest classification (R.F.), as well as of the human annotation is given.

The accuracy is much better for those foci where a prominence shift is predicted, that is, where the last accent does not fall on the last name ( $>40 \%)$. The accuracy for broad focus and third focus is substantially lower $(<31 \%)$. The annotator also was susceptible to this, and often confused broad and third focus, showing a strong bias to annotate third focus over broad focus. For the interrogatives, the accuracy for all foci is much lower $(<30 \%)$, and close to chance.

Fig. 8 shows the ranking of the importance of the predictors based on the permutation score on out-of-bag accuracy. The solid black line shows zero-a predictor of an importance score close to zero does not contribute much to the accuracy of the model. The dashed line plots the absolute value of the lowest negative predictor. Any predictor to the right of this line plausibly contributes to the model, while predictors to the left probably do not: Since non-informative predictors will have random importance scores fluctuating around zero, the absolute value of the lowest predictor is often taken as an approximate cut-off to distinguish predictors that contribute information and predictors that do not (Strobl et al., 2009). $\mathrm{F}_{0}$ of the initial syllable and duration of the first and final syllable appear to be the best cues to focus in the declarative data.

For the interrogative data, random forests are roughly at chance, and it seems that focus is simply not systematically encoded. 

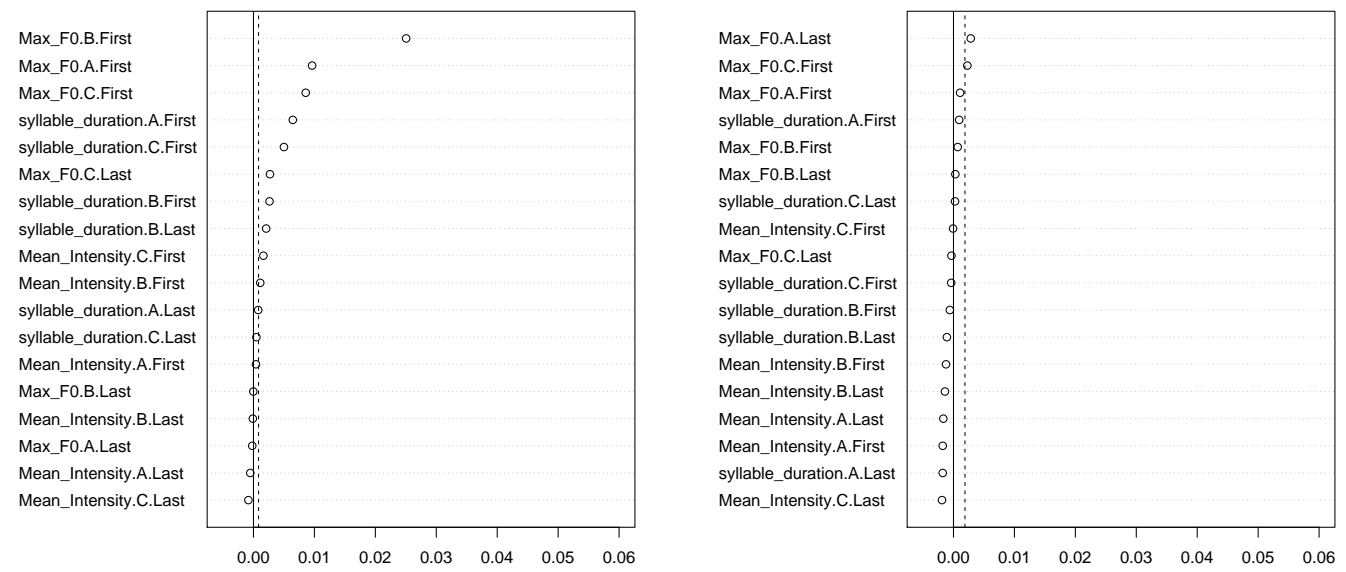

Figure 8: Importance of variables in random forest classification of Focus for the Declarative (left) and the Interrogative (right) utterances respectively

Having established the cues to focus prominence, let's now look at phrasing. Again we applied the random forest analysis on three different sets of data: the entire data set, the subset only consisting of broad focus, and the subset only consisting of focus on the first constituent and for which the annotators were able to hear first focus correctly. The latter data set is the one where we should see information about phrasing lost if phrasing cues are indeed adversely affected in the post-focal domain. The accuracy of the out-of-bag predictions are plotted in Table 5.

\begin{tabular}{lllll}
\hline & Constituency & R.F./Annotator-All & R.F./Annotator-Broad & R.F./Annotator-First \\
\hline 1 & (AB)C & $0.79 / 0.61$ & $0.75 / 0.63$ & $0.74 / 0.6$ \\
2 & A(BC) & $0.74 / 0.62$ & $0.74 / 0.61$ & $0.79 / 0.63$ \\
\hline
\end{tabular}

Table 5: Proportion of accurate classification for all data, broad focus data, and first focus data. For each data set, both the accuracy of the random forest classification (R.F.) and the human annotation is given. 
The results show that the RF classification in all three data sets was comparable: In the classification on the left-out data they all performed at or above $74 \%$ accuracy (where chance is 50\%). Crucially the classification worked just as well for the data set with initial focus. The human annotators performed more poorly than the RF model, but still better than chance, at an accuracy of 0.6 or above. Again, the accuracy of branching was not lower in the data set with initial focus.

Fig. 9 shows the relative importance of the predictors for the classification of phrasing. For both broad and first focus data, the same acoustic features did most of the work, namely the duration and intensity of the final syllable. There is also some evidence that the pitch of the accented initial syllable is relevant, but it only makes a small contribution to the classification, and the importance of this measure drops in the post-focal domain.
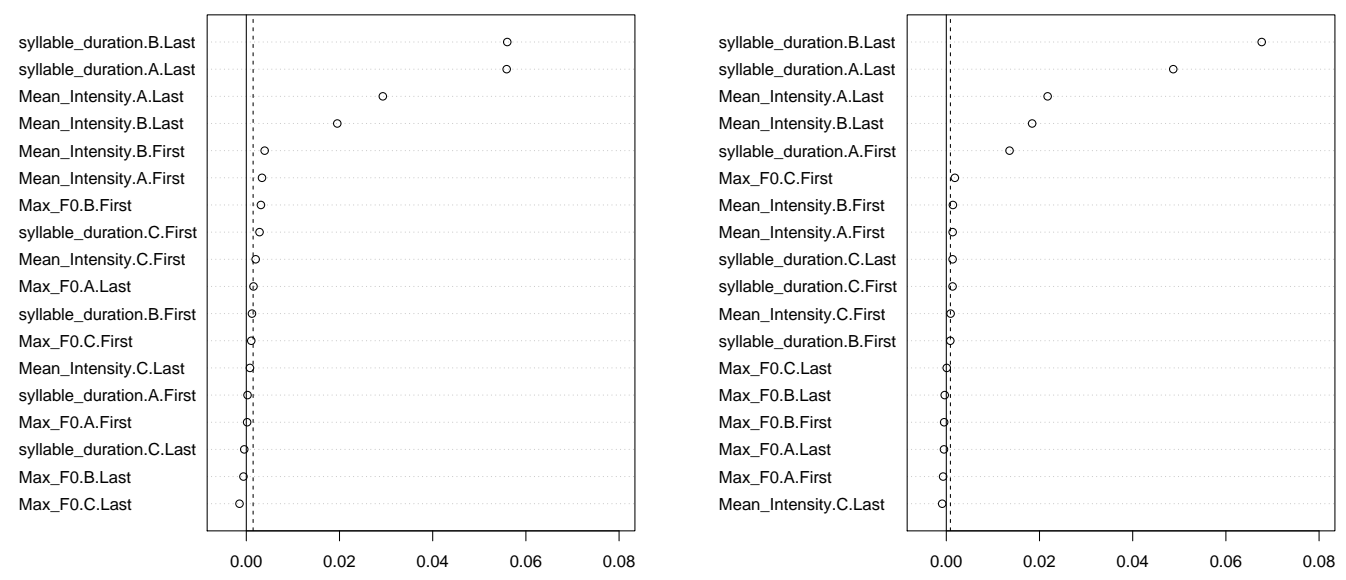

Figure 9: Importance of variables in random forest classification of Constituency for the case of broad focus (left) and first focus (right) respectively 


\subsection{Discussion}

The random forest analysis for focus confirmed what we already anticipated given the empirical plots and the regression models: Focus was cued less reliably in interrogatives. The random forest analysis added further information, however, regarding how well the information was cued. For the most part, it seems that only first focus was encoded with any success in the interrogative condition, and even in that condition it was close to chance. The human annotators were not much better than the random forest models, which suggests that we didn't simply feed the model the wrong acoustic measures.

These results are somewhat surprising, since it is clearly possible in principle to mark focus in questions. For example, focus is clearly conveyed in the interrogative utterances we used for illustration in 4 . Based on our own perception of the data, it seems to us that in the interrogative condition, speakers often simply didn't bother to mark focus. This may show that focus marking in interrogatives is less obligatory than in declaratives. Given the high correlation of tune and function, it is not clear whether this is due to the different tunes themselves, or of the fact that the utterance is (semantically) a question vs. a declarative. If it is the former, then this would be a case of tune-choice affecting focus marking, related to observations about such interactions in Goodhue et al. (2016) and Schlöder (2018). The latter possibility could maybe relate to the fact that a question resets the question under discussion, and maybe then there is less pressure to acknowledge prior focus antecedents. It could also be, however, that realizing focus is simply more difficult in questions for phonological or phonetic reasons, and hence speakers avoid marking it. This clearly merits further study. For example, it would be interesting to test whether failing to mark focus prosodically leads to similar degrees 
of infelicity in perception as omitting to mark focus in declaratives.

Another interesting finding in the focus models is that first and second focus are cued more reliably than the distinction between third and broad focus. These are the two conditions where there is a 'prominence shift', that is, the last accent falls on a different word than in the broad focus condition. This supports the view that relative prominence is at the heart of marking focus in English. Focus was easier to classify whenever relative prominence relations where changed. Both random forests and the hand annotations show that the prosodic cues that differentiate broad and third focus are not as strong as those that identify the first and second focus. Our results hence confirm earlier findings that broad and narrow focus tend to be confusable in English, unless there is a prominence shift to an earlier word-even though there are subtle acoustic cues that differentiate them (Gussenhoven, 1983). Similar findings have been reported for other languages (Botinis et al., 1999; Xu et al., 2012) suggesting that final focus and broad focus are phonetically (and perceptually) similar more generally. We already saw a related asymmetry in the pitch plot in Fig. 7, in that pitch reduction appeared to be a more reliable cue post-focally than pre-focally.

This linear asymmetry provides evidence for a 'focus ambiguity' (a term introduced by Jacobs 1991) between broad and late focus. This ambiguity of late and broad focus was observed already in Chomsky (1971), and was called 'focus projection' in Höhle (1982). The fact that the classification is still better than chance suggests that this is not a categorical effect, in contrast to what would be expected by accounts that have proposed that late focus can literally 'project' and is indistinguishable from early focus, as is predicted by certain accounts (e.g. Selkirk, 1995) (see Arregi see 2016 for a review of this type of focus projection). See also 
Breen et al. (2010) for related findings.

There is an interesting difference between human annotator and random forest model. Both were likely to confuse late focus with broad focus. The human annotator showed a strong bias toward annotating third focus. The random forest model, on the other hand, showed more symmetric confusion patterns. We see further evidence that, in the absence of reliable prosodic cues, listeners have a bias toward assuming narrow focus on the final constituent in the annotation focus for the interrogative data: Our human annotator categorized many interrogative utterances as third focus even if they were not.

The random forest model of focus classification illustrates how this method can make apparent to what extent information about one dimension (here: focus) is lost given another dimension (here: type of speech act). Our main research question, however, was whether phrasing cues are maintained in the post-focal domain. If initial focus adversely affects cues to phrasing later in the utterance, then we should find that a random forest trying to classify phrasing should perform worse in the case of first focus, similar to the random forest for focus which fared worse in the case of interrogative utterances.

The results of the random forest analysis for phrasing show that phrasing information remains intact in the post-focal domain (see Table 5). There was only one sign of an effect of focus on phrasing, which is that the importance of fundamental frequency decreases somewhat in the post-focal domain. Remember, however, that our pitch measures were not significantly affected by phrasing to begin with. The plot of predictor importance in Fig. 9 confirms that pitch only plays a very minor role in cueing phrasing in this data, both in broad and first focus. Overall, the phrasing appears to be left entirely intact post-focally. 
One might object to the line of reasoning here as follows: Could it not be that many participants simply did not produce the utterances with initial focus, even though the context was designed for them to do so? And could it not be that the utterances accurately classified for phrasing were exactly those where focus was not marked? To address this concern, we fitted an additional random forest model on only those utterances in which first focus was correctly annotated (a total of 96 soundfiles), based on the same acoustic predictor variables. ${ }^{22}$ This model even classified $85 \%$ of the utterances correctly for phrasing for the out-of-bag predictions, so there is no indication that phrasing was cued less reliably when first focus was successfully conveyed. In fact, it seems as if phrasing was cued more successfully with first focus. However, this apparent improvement is probably due to selection bias: Someone who successfully conveyed focus was clearly paying attention to the task, and may hence also perform better on conveying phrasing. So while we should not take the improvement as being too meaningful, the high level of accuracy clearly shows that when focus prosody is successfully applied, phrasing is still detectable, confirming earlier evidence from Norcliffe and Jaeger (2005).

The random forest analyses also point to how listeners may accomplish the difficult task of disentangling the different dimensions from the signal: The information of different dimensions is often distributed to different syllables. This, in combination with the fact that the acoustic cues have different relationships with each other for the different dimensions, makes it possible to retrieve different

\footnotetext{
${ }^{22} \mathrm{~A}$ plot of the importance of the various acoustic cues for this model is included in the supplementary materials. The top four acoustic cues were the same as for the entire first focus data plotted in Table 9 .
} 
kinds of information from the signal.

\section{Focus Prominence vs. Phrasal Prominence}

The results of this study suggest that certain interactions between focus prominence and phrasing prosody that are standardly assumed in many AM-models of sentence prosody may not in fact be attested. The central finding is that early focus in a sentence does not neutralize phrasing-distinctions in the post-focal domain. Experimental data suggesting that post-focal phrasing is maintained in English are also discussed in Norcliffe and Jaeger (2005) (and Jun and Fougeron, 2000; Sugahara, 2003; Ishihara, 2003, 2016; Kügler and Féry, 2017, for other languages). The finding that phrasing cues in the post-focal domain are not just present, but cued just as as reliably, is new.

This result is expected under overlay models that assume that different functional dimensions independently affect the phonetic realization of an utterance, as they were first proposed in Öhmann (1967) and Fujisaki (1981). If more generally, the prosodic dimensions of prominence, phrasing, and tune contributed to the overall pattern in an additive way (just as their roughly corresponding functional dimensions, focus, constituent structure, and speech act are orthogonal to each other), this would bode well for models that view sentence prosody as being composed from separate functions that each independently contribute to the overall sentence prosody, such as the Penta model ( $\mathrm{Xu}, 2005)$, or the Superposition of Functional Contours Model (Bailly and Holm, 2005; Gerazov et al., 2018). But would this necessarily mean that we should adopt a more phonetic view of sentence prosody, and abandon a mediating metrical representation?

It seems to us that this would be too strong a conclusion to draw. There is 
evidence for a sequential organization of the tonal events in an intonation contour (Ladd, 2008), which speaks against certain overlay model such as Fujisaki (1981), in that they cannot account for the precise way in which tonal events align with the words in a sentence. This is hard to capture without a metrical representation $\sqrt{23}$ Moreover, it is not the case that there are no interactions between the different functional dimensions - rather, our main finding is that one particular interaction, the obliteration of post-focal phrasing, assumed in some prior accounts, is simply not attested. In the following, we will discuss what kind of phonological representation of sentence prosody could capture the observed pattern.

\subsection{Focus prominence and post-focal phrasing}

Many current approaches to sentence prosody assume syntactic/semantic functions such as speech act, constituent structure and focus exert their influence indirectly, mediated by a metrical phonological representation. Our results suggest that the representations of prosodic tune, prominence, and phrasing should be kept more separate than is often assumed. Most importantly, focus should leave the prosodic effects of constituent structure on phrasing largely intact. This means, for example, that the representation of initial focus should leave the representation of phrasing in the post-focal domain intact, such that the following two utterances are not neutralized:

a. First Focus, left-branching:

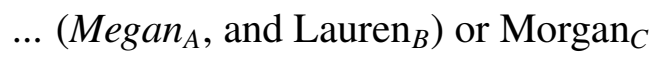

\footnotetext{
${ }^{23}$ Ladd (2008) furthermore discusses evidence for a mediation of phonetic reflexes by a phonological representation which may be a problem for more recent sequential overlay models as the ones in Möbius (1993) or Xu (2005), and speak in favor of a phonological mediation of phonetic effects. We refer the reader to Ladd (2008) for an extensive discussion.
} 
b. First Focus, right-branching:

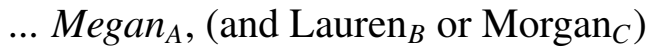

At least some metrical approaches, however, would predict a neutralization of (13a) and (13b), Consider again the pitch tracks in Fig. 3 and Fig. 4. We saw that with first focus, the fall of a declarative contour and the rise of a question contour starts immediately after the initial syllable (the one carrying the accent) of the focused word. According to the analysis of Pierrehumbert (1980) and others (e.g. Beckman, 1996), this would suggest that there are no intermediate or intonational boundaries after the focused constituent (other than at the end of the utterance). Both utterances in (13) should receive the same representation then, neutralizing the phrasing distinction: ${ }^{24}$

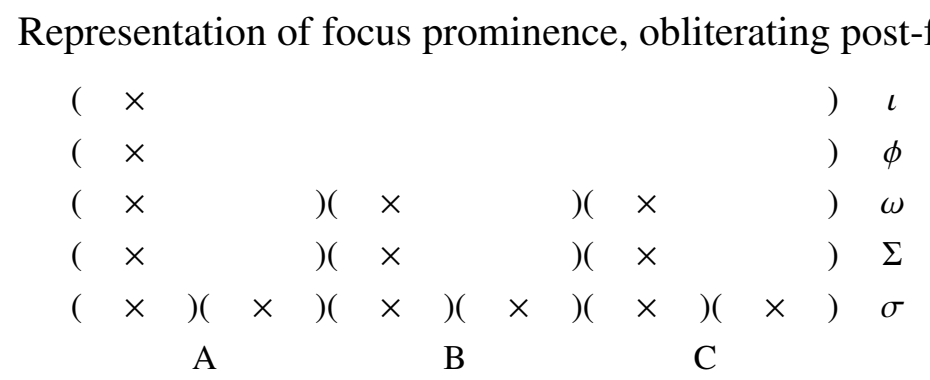

This representation of focus prominence is incompatible with our finding that post-focal phrasing is realized just as reliably as in the control case with broad focus.

Truckenbrodt (1995), however, proposes a metrical theory of sentence prosody

\footnotetext{
${ }^{24} \mathrm{We}$ will illustrate the idea based on the hierarchy assumed in Féry (2013), but added the lower prosodic levels ofthe foot $(\Sigma)$ and the syllable $(\sigma)$ here to be able to refer to different syllables within a word.
} 
in which maintaining phrasing distinctions post-focally is an option. In his account, if the alignment constraints responsible for syntax-induced alignment are highly ranked, focus-alignment can be achieved while maintaining more phrasing information post-focally:

(15) Representation of focus prominence, leaving phrasing intact:

a. Left-Branching, initial focus:

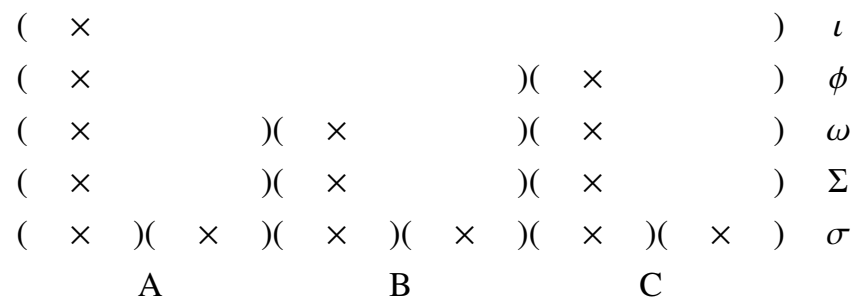

b. Right-branching, initial focus:

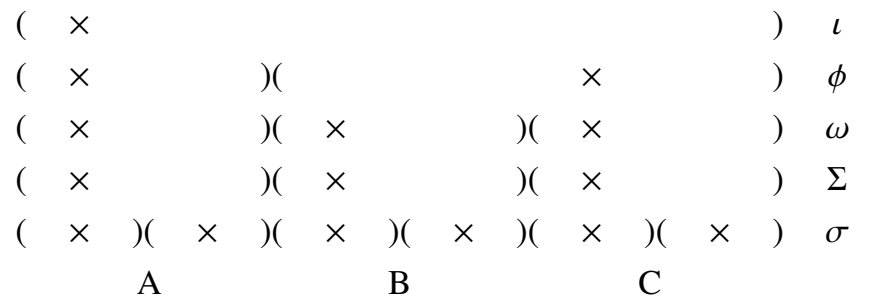

This representation encodes focus prominence by shifting which syllable projects highest at the intonational phrase level, while leaving lower level phrasing distinctions at the phonological (or intermediate) phrase-level intact. Note that in this theory, prosodic constituents are assumed to be left-headed up to the wordlevel and right-headed above the word-level (starting with $\phi$ ). Focus can override the headed-ness in higher level constituents, enforcing early focused constituents to become the projecting head. This type of representation is compatible with the findings that post-focal phrasing is maintained. A representation of sentence prosody that allows for the preservation of post-focal phrasing by focus-driven 
changes in headedness is assumed in various current accounts (Truckenbrodt, 1995; Büring, 2010a; Féry and Ishihara, 2009; Féry, 2013; Kügler and Féry, 2017, i.a.).

There are other representational options, however. Jun (2011) reports evidence that in Korean too, phrasing can still be encoded post-focally, a phenomenon referred to as 'phonetic dephrasing' in this work. In order to account for phonetic dephrasing, Jun (2011) posits the existence of deficient ('deformed') extra-prosodic phrases that can act as prosodic suffixes (for reduced post-focus material) or prefixes (for reduced pre-focal material) to intonational phrases or even intermediate phrases. It is not clear, however, whether there is any independent evidence for such additional types of deficient prosodic units.

The representation in (15) illustrates that in all of these approaches, the height of projection in the grid is influenced both by phrasing (since only one syllable within each phrase will project to the next highest level) and by focus (since one syllable of the focused word must project highest). The underlying idea is that perceptual prominence, which is influenced by both of these factors, can be uniformly represented by grid projection height. Let's consider the way prominence is influenced by phrasing under this set of assumptions in more detail, and assess how well this fits with our results.

\subsection{Phrasing-related prominence}

Each prosodic constituent in the prosodic hierarchy is typically assumed to have exactly one grid mark at its top line (the 'head' of that prosodic constituent). Prosodic phrasing thereby directly affects how high a syllable is projected on the grid, just as focus does. This is meant to capture phrasing-related intuitions about prominence, as well as helping to explain where tonal targets will be realized 
within a phrase. Let's consider the two phrasings we observed in our broad focus conditions. Our experimental results show evidence that the prosodic phrasing within the coordinate structure is as follows (where ',' indicates the intended syntactic constituent structure and '(..)' the prosodic constituency)

a. Broad focus, right-branching:

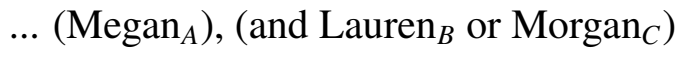

b. Broad focus, left-branching:

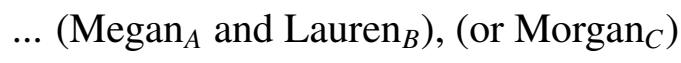

There is a clear intuition that Lauren is more prominent in (16b) than in (16a). Moreover, there is an intuition that Lauren is more prominent than Megan in (16b), while it is less prominent than Megan in (16a). In other words, not just focus, but also prosodic phrasing affects our intuitions about relative prominence. In fact, some early approaches to the metrical grid sometimes represented phrasing and grouping purely by using prominence marks in an unlabeled metrical grid (e.g. Prince, 1983). Approaches such as Truckenbrodt (1995) also encode phrasingrelated prominence by projection-height in the grid, the very same tool also used to encode focus prominence. The representations of the two structures in (16) would be the following:

Representation of phrasing with headed prosodic constituents

\footnotetext{
${ }^{25}$ The prosodic affiliation of the connectors 'and' and 'or' are sometimes not entirely clear. It is very possible that as function words they are able to encliticize to the preceding prosodic domain instead of being phrased with the following conjunct.
} 
a. Left-Branching, broad focus:

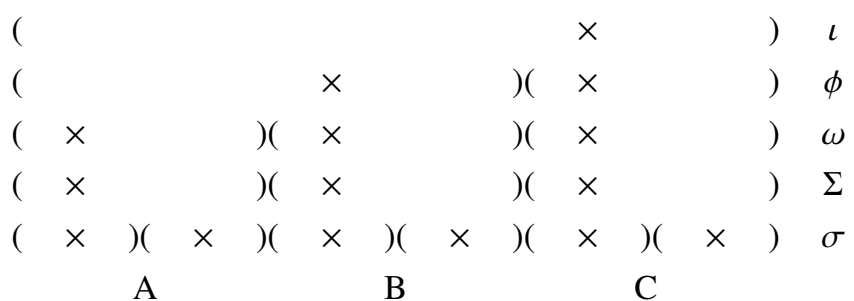

b. Right-branching, broad focus:

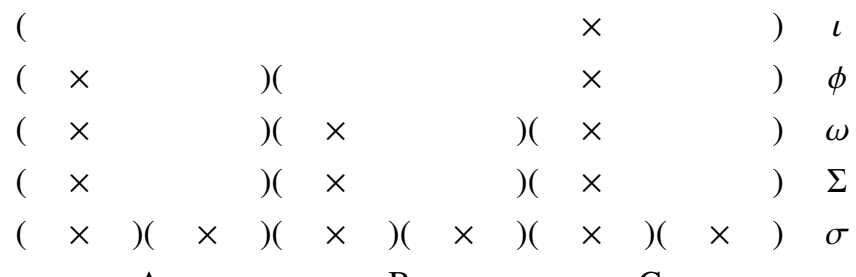

A $\quad$ B $\quad$ C

There is good empirical evidence that phrasing indeed affects perceptual prominence. For example, recent studies using Rapid Prosodic Transcription (Cole et al. 2010) has provided evidence that naïve listeners' intuitions about the prominence of a constituent are affected by the position within a phrase it occurs in, such that accented constituents at the end of phrases are perceived as more prominent than those phrase-internally (see also Bishop et al., this special issue; Cole et al., 2019 this Special Issue, ${ }^{26}$

Our results raise some questions, however, whether it is indeed accurate to represent phrasal prominence and focal prominence in the same way.

\subsection{Phrasal prominence uses different cues}

Let's consider the acoustic cues that encode phrasing-related prominence. In (16b), where Lauren is phrase-final and is intuitively more prominent than Megan,

\footnotetext{
${ }^{26}$ See Vainio and Järvikivi $\sqrt{2006)}$ for related findings in Finnish.
} 
the intensity of both initial syllable and final syllable is lower, compared to (16a), where it is phase-initial (see Fig. 6, and Table 2).

Focus prominence is realized differently, however. Let's consider the prominence relations in the following two utterances:

\section{a. First Focus:}

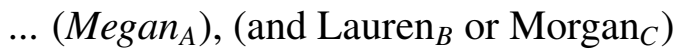

b. Second Focus:

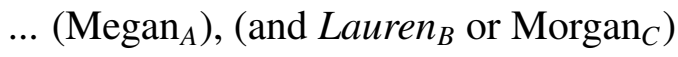

The word Lauren is intuitively more prominent when it is focused (as in (18b)) compared to when the first word is focused (as in (18a). But this time, the intensity of the accented syllable of Lauren is significantly higher when it is more prominent (see Fig. 6; and Table 2). So the phonetic cues for phrasing-prominence and focus-prominence are quite different.

One could try to account for these differences by associating different acoustic effects with grid-marks at different levels in the representation: ${ }^{27}$ Maybe grid marks at the $\phi$-level induce greater duration in the following syllable (final lengthening) while grid marks at the $\iota$-level induce greater $F_{0}$ and intensity. If focus then affects projection to the $\iota$-level and syntax projection to the $\phi$-level, then the difference in the cues observed for focus-prominence and phrasing-prominence could be accounted for. This set of assumptions fails to capture some of the generalizations in our data, however. In the broad focus condition, why does the last (or 'nuclear') accent, which projects to the $\iota$-level, not receive higher intensity

\footnotetext{
${ }^{27}$ Thanks to the editors for suggesting this interpretation of the effects.
} 
than other accents - unlike material that projects to the $\iota$-level for focus reasons? Why is less prominent, non-focal material (which does not project to the $\iota$-level) marked with lower pitch in declaratives and higher pitch in questions when it is less prominent for focus reasons, but not when it is less prominent for reasons of phrasal prominence (where it should also not project to the $\iota$-level)? Why do utterance-final words show less final lengthening than pre-boundary material that is not utterance-final (Wagner, 2005)? Maybe the assumption that focusprominence and phrasing-prominence are represented in the same way should be reconsidered.

\subsection{Dissociating Phrasing Prominence and Focus Prominence?}

Wagner (2005) proposed a representation that abandons the idea that our percept of relative prominence will map uniformly to projection-height. The prosodic representation proposed instead represents focus-prominence and phrasingprominence in different ways. The proposed metrical representation consists of an unlabeled metrical grid (that is, a metrical grid in which the lines are not labeled with prosodic categories like $\iota$ or $\phi$, as is usually assumed elsewhere). Constituents that are at the same syntactic 'level' are mapped to equal prosodic domains on a grid line $\sqrt{28}$ The representation crucially differs from alternatives in that in a given prosodic domain, there does not need to be a single grid mark that projects highest:

$$
\text { Representation of prosodic phrasing according to Wagner 2005, 2010): }
$$

\footnotetext{
${ }^{28}$ Syntactic levels are defined as all constituents having been merged within the same cycle or 'phase', details will not be discussed here.
} 
a. Left-Branching, broad focus:

b. Right-branching, broad focus:

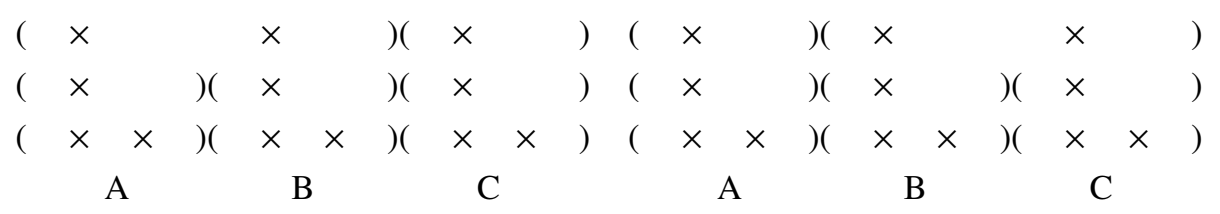

The metrical grid encodes grouping by virtue of boundaries of different strength, where boundary strength correlates with the line in the grid at which a boundary is represented. Note that the representation does not directly represent why Lauren (=B) should be more prominent than Megan (=A) in (a), but less prominent in (b). Rather, all three names project to the highest grid line, and as a result will all be associated with a pitch accent. This analysis treats the phrasing-related intuitions about differences in prominence between the names as an indirect consequence of phrasing, due to a perceptual principle that has the effect that the last in a series of equals will be perceived as more 'prominent' or 'salient'.

This idea goes back to Newman (1946), who proposed that the 'nuclear stress' is simply the last among equal accents within a domain. What's new in the presentation here is that prior accounts always assumed that the nuclear stress is singled out representationally (e.g. Chomsky and Halle, 1968; Truckenbrodt, 1995). Leaving it representationally at the same level, however, seems more compatible with the observation that the final accent is not phonetically special. For example, Pierrehumbert (1979) showed that final accents are perceived as more prominent despite their lower $\mathrm{F}_{0}$ (a result of declination and downstep), and lower intensity (a result of intensity down-drift). In addition, Liberman and Pierrehumbert (1984) found that the $F_{0}$ of final accented words is in fact lower even than would be expected based on declination and downstep effects, and label this effect 'final lowering'. From a purely acoustic point of view, there is no sign then that the 
nuclear accent should be 'prominent'. The reason it is still perceptually the most prominent nevertheless, is not to be found in the acoustics. We 'get away' with allocating less intensity and lower pitch to it, arguably due to a perceptual principle that has the effect that final constituents are inherently salient. So maybe it is not necessary to encode the special status of the last prominence within a domain in the metric representation-it is singled out already by being last. ${ }^{29}$

While phrasal prominence is simply represented as 'last among equals' in Wagner (2005), focus prominence (as well as word stress) is represented via grid height, similar to the standard account. The prominence of focused constituents and the reduction of given constituents comes about through 'prosodic subordination', where certain constituents project higher due to focus while others that do not are thereby subordinated. Wagner (2005). This process of subordination leaves phrasing intact 30 Representation of focus prominence according to Wagner (2005, 2010):

\footnotetext{
${ }^{29} \mathrm{We}$ note that it is not the case that naive listeners will necessarily pick out the word carrying nuclear stress when asked which is the most prominent word. The notion 'prominent' is not selfexplanatory. Trained annotators, however, such as our two annotators, show a high correlation annotating where the nuclear accent falls. Also, naive speakers will align the high tone of the vocative chant with the nuclear accent. This shows that the notion 'nuclear stress' is real, even if it is not trivial to define it in terms of prominence.

${ }^{30}$ Prosodic subordination is similar in effect to the 'swap' discussed in Büring (2010b), where in a given line on the grid the unique mark that projects to be the head with a domain can be changed or 'swapped', such that an F-marked constituent projects and the one that should have projected by default does not. In Wagner (2005), instead of 'swapping' which grid mark becomes the head, it is the grid mark(s) on the constituent bearing an F-marker that projects higher up, while the remainder remain untouched. Note that the precise grid-distribution in Wagner (2005) is slightly different due to the way the metrical tree is derived recursively from the syntactic structure, by computing relative prominence between sister constituent from inside out. See Büring (2016) for arguments in favor of a sister-based algorithm similar to the one proposed in Wagner (2005).
} 
a. Left-Branching, initial focus:

b. Right-branching, initial focus:

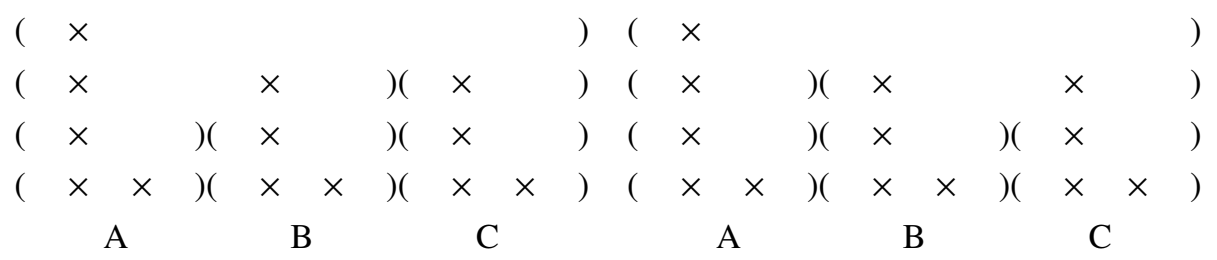

In this metrical representation, focus and phrasing affect the phonological representation in rather different ways, which both contribute to the percept of prominence. Both the height of projection (which is affected by focus, among other factors), and being last in a sequence of equal grid marks will contribute to the perceived prominence of a constituent. And these are just two out of potentially many factors that may affect perceived prominence. Cole et al. (2010), for example, proposes that word frequency independently affects prominence judgments (and affects the acoustics of words in a different way compared to other factors), an effect replicated in Bishop et al. (this special issue) 3

If we dissociate the representation of phrasal effects on prominence from focal effects of prominence in this way, then we no longer expect that the phonetic correlates of phrasal prominence and focus prominence will be the same-even if they both influence perceptual prominence 32

How then do we get from the unlabeled metrical grids in (19) and (20) to the acoustic realization of the utterance? The representation allows us to state general-

\footnotetext{
${ }^{31}$ Note also that in this account, no assumptions about right-headedness of prosodic constituents above the word level have to be made.

${ }^{32}$ Note that this view does not sit well with the idea in Büring (2010a) and Féry (2013) that languages differ in whether they use focus prominence as defined here or instead exploit phrasal prominence to 'align' focus with prominent positions in the sentence. But we note that it is not clear that languages that encode focus purely via phrasing really mark the same focus presupposition as focus prominence does in languages like English and German. Discussing this in detail would go beyond the scope of this paper.
} 
izations about duration an intensity as follows: Vowels that project to higher grid lines will receive higher intensity and duration than vowels projecting to lower grid lines (this is how focal prominence comes about); vowels associated with a mark on a given grid line will receive decreasing amounts of intensity compared to earlier vowels that project to the same level ('down drift'); and intensity is increased after an initial '('-boundary ('initial strengthening'); vowels that precede a '(' boundary are lengthened proportional to the grid line at which the '('- boundary occurs ('pre-boundary lengthening'). Crucially, a word that is more prominent than another word can either have higher intensity (if its greater prominence is due to focus prominence) or lower intensity (if its greater prominence is due to phrasal prominence) ${ }^{33}$

How do the phonological events associated with a particular tune, such as pitch accents and boundary tones, get associated with the right vowels, given a representation that does not identify which level of the grid corresponds to the intonational phrase? Liberman (1975) and Pierrehumbert (1980) already presented frameworks in which tone association rules simply make reference to the height of grid columns, such that all grid marks projecting to the top grid line are associated with pitch accents, whose identity depends on the overall tune that was chosen for an utterance. Focus-induced subordination, in combination with the convention that only syllables with grid marks that project highest will receive a pitch accent, will have the effect that focused words will be accented, and material after the focus will not be. This will result in focused words being more prominent com-

\footnotetext{
33 Note that to state these generalizations on the left parentheses of the grid ('(') need to be referred to, we could omit ')' altogether, following ideas in Idsardi(1992). This is one reason why Wagner (2005) actually used pipe-symbols instead of the directed parentheses used here. Pitch scaling effects can also in principle be stated in relation to the grid (see Wagner, 2005, 140ff).
} 
pared to non-focused material. Additional association rules are needed that relate boundary tones to boundaries in the grid.

One distinguishing feature of this representation is that not every utterance has equally many grid lines above the word-level. Single-word utterances might have none, and most sentences might only have one or two grid lines above the one corresponding to the word, depending on the syntactic structure of the utterance. The metrical presentation is hence more parsimonious than the one standardly assumed. Gussenhoven (1990) and Gussenhoven and Rietveld (1992) also proposed a model of tonal association that is compatible with this idea.

The proposed representation is compatible with at least some observations about the placement of 'phrase accents'. These are the tonal events following the pitch accents of a tune, which are usually assumed to be tied intermediate phrases. However, their distribution could be characterized instead in reference to secondary prominences (lower grid marks) in the metrical grid. For example, the final mid-level tone of the vocative chant in American English appears to sometimes align with an unstressed syllable directly after the pitch accented syllable (for example when the utterance just consists of a bisyllabic name with initial stress), and sometimes with a post-nuclear secondary stress (whenever there is a syllable carrying stress following the nuclear stress) (cf. Liberman, 1975; Grice et al., 2000). This phrase accent might simply associate with the first grid mark of the next level down after the nuclear stress (the last grid mark on the top line) (see Wagner, 2005, 153ff). It remains to be seen whether the metrical representation assumed here is compatible with all alignment patterns of phrase accents observed in the literature (e.g. Grice et al., 2000; Barnes et al., 2010).

In light of our experimental findings, a dissociation of the phonological rep- 
resentation of phrasal prominence and focus prominence seems desirable then. We acknowledge, however, that there is also some indication that we should not completely dissociate these notions. For example, the errors that annotators made for the broad focus condition suggest that they confounded focus prominence and phrasing prominence, as illustrated in Table 6 .

\begin{tabular}{llrrrr}
\hline & Constituency & Broad & First & Second & Third \\
\hline 1 & (AB)C & 28.00 & 2.00 & 12.00 & 69.00 \\
2 & A(BC) & 43.00 & 11.00 & 6.00 & 60.00 \\
\hline
\end{tabular}

Table 6: Confusion matrix for the annotation of Broad Focus utterances depending on phrasing

Most of the wrong classifications of focus in constituent A and B involve cases in which they had phrasing prominence, i.e., they were wrongly annotated as focused in a case were they were final in a prosodic domain. This suggests that at least in perception, focus prominence and phrasing prominence are confusable.

It is also conceivable that just looking at the discrepancies in the phonetic cues in the way we have done is simply misleading. Maybe if we looked at the data in a different way, there would be a greater uniformity in how the two kinds of prominence are cued. Suppose, for example, that the 'real' cue to prominence is the number of pitch pulses on a word (i.e., the product of the $F_{0}$ frequency and the duration), or the total energy exerted (i.e., the area under the intensity curve, which combines the effects of intensity and lengthening). Such aggregate measures could in principle lead to a greater uniformity of the cues for the two kinds of prominence. We added plots of these two types of measures in the supplementary materials. They qualitatively look very similar to the duration plots, however, and therefore fail to create a more uniform pattern for focal and phrasal prominence. However, maybe we simply haven't thought yet of the right dependent 
measure that would accomplish this ${ }^{34}$

\section{Conclusion}

An experiment looking at interactions between different functional dimensions (focus, constituency, speech act) provided evidence that changes in the location of focus mainly affects the prosodic prominence pattern of an utterance, and leaves its prosodic phrasing intact. More specifically, placing early focus does not neutralize phrasing distinctions in the post-focal domain. This speaks against some theories of sentence prosody that predict such a neutralization. The results speak in favor of a representation that does not conflate focus-related effects on prominence with constituent-structure-related effects on phrasing. We also found that the phonetic cues to phrasing-related prominence are substantially different from the cues for focus-related prominence. This provides evidence that focusrelated effects on prominence should be represented differently from constituentstructure-related effects on prominence.

We discussed one theory of metrical prosody compatible with these findings, which disentangles phrasing and prominence, the metrical grid proposed in Wagner (2005). In this representation, focus affects prominence by changing the height at which certain constituents project on a metrical grid, while phrasing only affects the placement of metrical boundaries. Phrasing-related effects on perceived prominence are attributed to the salience of final constituents over non-final domains within a domain, rather than representing them (like focus) by projectionheight.

\footnotetext{
${ }^{34} \mathrm{~A}$ promising measure we have not considered yet is the combination of $\mathrm{F} 0$ information with the periodic energy curve (Albert et al., 2018, Cangemi et al., 2019).
} 
An interesting side-result of our study is that intensity appears to be better cue to phrasing than $\mathrm{F}_{0}$ scaling. The observed phrasing-related intensity pattern goes beyond the intensity 'downdrift' already observed in Pierrehumbert (1979). Given the inherent link between intensity and $\mathrm{F}_{0}$, we observed that this pattern raises the possibility that some apparent $\mathrm{F}_{0}$ correlates of phrasing, for example pitch-accent scaling, might actually be an indirect consequence of a primary manipulation of intensity to encode phrasing. More research will be needed to explore this result. It remains to be seen, for example, whether this is also perceptually an important cue to phrasing.

It is clear that our discussion of the two types of prominence, and of the proper representation of sentence prosody, remains very preliminary. Both the metrical representation we favored here and the more standard representation based on the prosodic hierarchy fail to do justice to the fact that prosody is produced incrementally in real time. Any static representation of sentence prosody may miss generalizations that only make sense from an incremental point of view, and once the locality of production planning (cf. Keating and Shattuck-Hufnagel, 2002) and the dynamics of articulating in real time are taken into account (cf. Mücke et al. 2014). Whichever approach to sentence prosody we take, we will have to account for the fact that the prosodic effects of focus and those of constituent structure are (largely) orthogonal- even if their effect on perceived prosodic prominence is not.

\section{Acknowledgements}

We would like to thank the reviewers and the two editors, Stefan Baumann and Francesco Cangemi, for the many helpful comments they provided. We would 
also like to thank Daniel Asherov, Jennifer Cole, Meghan Clayards, Morgan Sonderegger, and Francisco Torreira for helpful comments on this project. Thanks to the members of prosody.lab for help with running the study and annotating the data, especially David Fleischer, Thea Knowles, and Erin Olson, as well as to Sonora Grimsted for her help proofreading the article. This work was supported by SSHRC Grant 435-2014-1504, as well as by funding through the SSHRC Canada Research Chair program. The data this paper is based on is available at https://github.com/prosodylab/ArticleFocusPhrasingJphon.

\section{References}

Albert, A., Cangemi, F., and Grice, M. (2018). Using periodic energy to enrich acoustic representations of pitch in speech: A demonstration. In Proceedings of the 9th International Conference on Speech Prosody, pages 804-808.

Arregi, K. (2016). Focus projection. In Féry, C. and Ishihara, S., editors, The Oxford Handbook of Information Structure. Oxford University Press.

Bailly, G. and Holm, B. (2005). Sfc: a trainable prosodic model. Speech communication, 46(3-4):348-364.

Barnes, J., Veilleux, N., Brugos, A., and Shattuck-Hufnagel, S. (2010). Turning points, tonal targets, and the english 1-phrase accent. Language and Cognitive Processes, 25(7-9):982-1023.

Baumann, S. (2016). Second occurrence focus. In Féry, C. and Ishihara, S., editors, The Oxford Handbook of Information Structure. Oxford University Press. 
Baumann, S. and Winter, B. (2018). What makes a word prominent? predicting untrained german listeners' perceptual judgments. Journal of Phonetics, 70:2038.

Beckman, M. (1996). The Parsing of Prosody. Language and Cognitive Processes, 19(11):17-67.

Beckman, M. and Edwards, J. (1992). Intonational categories and the articulatory control of duration. In Tohkura, Y., Vatikiotis-Bateson, E., and Sagisaka, Y., editors, Speech perception, production and linguistic structure, pages 359-375. Ohmsha, Tokyo.

Beckman, M. E. and Ayers Elam, G. (1997). Guidelines for ToBI labelling. Ohio State University.

Beckman, M. E., Hirschberg, J., and Shattuck-Hufnagel, S. (2005). The orginal ToBI system and the evolution of the tobi framework. In Jun, S.-A., editor, Prosodic models and transcription: Towards prosodic typology, pages 9-54. Oxford University Press, Oxford.

Bishop, J., Kuo, G., and Kim, B. (this special issue). Phonology, phonetics, and signal-extrinsic factors in the perception of prosodic prominence: Evidence from rapid prosody transcription. Journal of Phonetics.

Björklund, S. and Sundberg, J. (2016). Relationship between subglottal pressure and sound pressure level in untrained voices. Journal of Voice, 30(1):15-20.

Botinis, A., Fourakis, M., and Gawronska, B. (1999). Focus identification in English, Greek and Swedish. In Proceedings of The 14th International Congress of Phonetic Sciences, San Francisco, pages 1557-1560. 
Breen, M., Fedorenko, E., Wagner, M., and Gibson, E. (2010). Acoustic correlates of information structure. Language and Cognitive Processes, 25(7):1044-1098.

Breiman, L. (2001). Random forests. Machine learning, 45(1):5-32.

Breiman, L., Friedman, J. H., Olshen, R. A., and Stone, C. J. (1984). Classification and regression trees. Chapman \& Hall, New York.

Büring, D. (2010a). A Weak Theory of Strong Readings. Ms., Cologne University.

Büring, D. (2010b). Towards a typology of focus realization. In Zimmermann, M. and Féry, C., editors, Information structure. Theoretical, typological, and experimental perspectives, pages 177-205. Oxford University Press, Oxford.

Büring, D. (2016). Intonation and meaning. Oxford University Press.

Byrd, D. and Saltzman, E. (1998). Intragestural dynamics of multiple phrasal boundaries. Journal of Phonetics, 26:173-199.

Byrd, D. and Saltzman, E. (2003). The elastic phrase: Modeling the dynamics of boundary-adjacent lengthening. Journal of Phonetics, 31:149-180.

Cangemi, F., Albert, A., and Grice, M. (2019). Modelling intonation: Beyond segments and tonal targets. In Proceedings 19th ICPhS, Melbourne, Australia., pages $572-576$.

Cho, T. (2002). The Effects of Prosody on Articulation in English. Routledge, New York.

Cho, T. (2016). Prosodic boundary strengthening in the phonetics-prosody interface. Language and Linguistics Compass, 10(3):120-141. 
Cho, T. and Keating, P. (2001). Articulatory and acoustic studies on domain-initial strengthening in Korean. Journal of Phonetics, 29(2):155-190.

Cho, T. and Keating, P. (2009). Effects of initial position versus prominence in english. Journal of Phonetics, 37(4):466-485.

Cho, T., Lee, Y., and Kim, S. (2011). Communicatively driven versus prosodically driven hyper-articulation in korean. Journal of Phonetics, 39(3):344-361.

Cho, T., McQueen, J., and Cox, E. (2007). Prosodically driven phonetic detail in speech processing: The case of domain-initial strengthening in English. Journal of Phonetics, 35(2):210-243.

Chomsky, N. (1971). Deep structure, surface structure, and semantic interpretation. In Steinberg, D. D. and Jakobovits, L. A., editors, Semantics: An Interdisciplinary Reader in Philosophy, Linguistics, and Psychology, pages 183-216. Cambridge University Press, Cambridge.

Chomsky, N. and Halle, M. (1968). The sound pattern of English. Harper \& Row, New York.

Cole, J., Hualde, J. I., Smith, C. L., Eager, C., Mahrt, T., and Napoleão de Souza, R. (2019, this Special Issue). Sound, structure and meaning: The bases of prominence ratings in English, French and Spanish. Journal of Phonetics, 75:113-147.

Cole, J., Mo, Y., and Hasegawa-Johnson, M. (2010). Signal-based and expectation-based factors in the perception of prosodic prominence. Laboratory Phonology, 1(2):425-452. 
Cooper, W. E., Eady, S. J., and Mueller, P. R. (1985). Acoustical aspects of contrastive stress in question-answer contexts. Journal of the Acoustical Society of America, 77(6):2142-2156.

Eady, S., Cooper, W., Klouda, G., Mueller, P., and Lotts, D. (1986). Acoustical characteristics of sentential focus: narrow vs. broad and single vs. dual focus environments. Language and Speech, 29(3):233-251.

Eady, S. J. and Cooper, W. E. (1986). Speech intonation and focus location in matched statements. Journal of the Acoustical Society of America, 80(2):402415 .

Edwards, J., Beckman, M., and Fletcher, J. (1991). The articulatory kinematics of final lengthening. The Journal of the Acoustical Society of America, 89:369.

Feldhausen, I. (2016). The relation between prosody and syntax: The case of different types of left-dislocations in spanish. In Armstrong, M., Henriksen, N., and del Mar Vanrell, M., editors, Intonational Grammar in Ibero-Romance: Approaches across linguistic subfields, pages 153-180. John Benjamins, Amsterdam.

Féry, C. (2010). German sentence accents and embedded prosodic phrases. MS. Potsdam University.

Féry, C. (2013). Focus as prosodic alignment. Natural Language $\mathcal{E}$ Linguistic Theory, 31(3):683-734.

Féry, C. and Ishihara, S. (2009). How focus and givenness shape prosody. In Zimmermann, M. and Féry, C., editors, Information Structure: Theoretical, 
Typological, and Experimental Perspectives, pages 36-63. Oxford University Press. Ms. Goethe Universität Frankfurt.

Féry, C. and Kentner, G. (2010). The prosody of embedded coordinations in German and Hindi. In Proceedings of Speech Prosody, volume 5.

Féry, C. and Truckenbrodt, H. (2005). Sisterhood and Tonal Scaling. Studia Linguistica, 59(2-3):223-243.

Fougeron, C. and Keating, P. (1997). Articulatory strengthening at edges of prosodic domains. Journal of the Acoustical Society of America, 101(6):37283740.

Fujisaki, H. (1981). Dynamic characteristics of voice fundamental frequency in speech and singing. acoustical analysis and physiological interpretations. KTH Department of Speech, Music and Hearing. Quarterly Status Report.

Gee, J. and Grosjean, F. (1983). Performance structures: A psycholinguistic appraisal. Cognitive Psychology, 15:411-458.

Gerazov, B., Bailly, G., Mohammed, O., Xu, Y., and Garner, P. N. (2018). A variational prosody model for mapping the context-sensitive variation of functional prosodic prototypes. arXiv preprint arXiv:1806.08685.

Goldman-Eisler, F. (1972). Pauses, clauses, sentences. Language and Speech, 15(2):103-13.

Gollrad, A. (2013). Prosodic cue weighting in sentence comprehension. $\mathrm{PhD}$ thesis, Universität Potsdam. 
Goodhue, D., Harrison, L., Su, Y. T. C., and Wagner, M. (2016). Toward a bestiary of English intonational tunes. In Hammerly, C. and Prickett, B., editors, Proceedings of the 46th Conference of the North Eastern Linguistic Society (NELS), Concordia University, pages 311-320.

Gramming, P., Sundberg, J., Ternström, S., Leanderson, R., and Perkins, W. H. (1988). Relationship between changes in voice pitch and loudness. Journal of Voice, 2(2):118-126.

Grice, M., Ladd, D. R., and Arvaniti, A. (2000). On the place of phrase accents in intonational phonology. Phonology, 17(2):143-185.

Grosjean, F. and Collins, M. (1979). Breathing, pausing, and reading. Phonetica, 36:98-114.

Gussenhoven, C. (1983). Testing the reality of focus domains. Language and Speech, 26:61-80.

Gussenhoven, C. (1990). Tonal association domains and the prosodic hierarchy in English. In Ramsaran, S., editor, Studies in the pronuncation of English. A commemorative volume in honour of A.C. Gimson, pages 27-37. Routledge, London.

Gussenhoven, C. (2007). Types of focus in English. In Lee, C., Gordon, M., and Büring, D., editors, Topic and Focus: Intonation and Meaning. Theoretical and Crosslinguistic Perspectives, pages 83-100. Kluwer, Dordrecht.

Gussenhoven, C. and Rietveld, A. C. M. (1992). Intonation contours, prosodic structure, and preboundary lengthening. Journal of Phonetics, 20:282-303. 
Hayes, B. and Lahiri, A. (1991). Bengali intonational phonology. Natural Language and Linguistic Theory, 9:47-96.

Hedberg, N., Sosa, J. M., and Görgülü, E. (2014). The meaning of intonation in yes-no questions in american English: A corpus study. Corpus Linguistics and Linguistic Theory.

Hedberg, N., Sosa, J. M., Görgülü, E., and Mameni, M. (2010). Prosody and pragmatics of wh-interrogatives. In Proceedings of the 2010 Annual Meeting of the Canadian Linguistics Society.

Höhle, T. (1982). Explikation für 'Normale Betonung' und 'Normale Wortstellung'. In Abaraham, W., editor, Satzglieder des Deutschen, pages 75-153. Narr.

Hothorn, T., Buehlmann, P., Dudoit, S., Molinaro, A., and Van Der Laan, M. (2006). Survival ensembles. Biostatistics, 7(3):355-373.

Idsardi, W. J. (1992). The computation of Prososdy. PhD thesis, MIT.

Ishihara, S. (2003). Intonation and Interface Conditions. PhD thesis, MIT.

Ishihara, S. (2007). Major phrase, focus phrase, multiple spell-out. The Linguistic Review, 24.

Ishihara, S. (2016). Japanese downstep revisited. Natural Language $\mathcal{E}$ Linguistic Theory, 34(4):1389-1443.

Jackendoff, R. S. (1972). Semantic Interpretation in Generative Grammar. MIT Press, Cambridge, Ma.

Jacobs, J. (1991). Focus ambiguities. Journal of Semantics, 8:1-36. 
Jun, S.-A. (1993). The phonetics and phonology of Korean prosody. PhD thesis, Ohio State University.

Jun, S.-A. (2011). Prosodic markings of complex np focus, syntax, and the pre/post-focus string. In Proceedings of the 28th West Coast Conference on Formal Linguistics, pages 214-230, Somerville, MA. Cascadilla Press.

Jun, S.-A. and Fougeron, C. (2000). A phonological model of French intonation. In Intonation, pages 209-242. Springer, Dordrecht.

Keating, P., Cho, T., Fougeron, C., and Hsu, C.-S. (2003). Domain-initial strengthening in four languages. In Local, J., Ogden, R., and Temple, R., editors, Phonetic Interpretation: Papers in Laboratory Phonology VI, pages 143-161. Cambridge University Press, Cambridge, UK.

Keating, P. and Shattuck-Hufnagel, S. (2002). A prosodic view of word form encoding for speech production. UCLA Working Papers in Phonetics, 101:112156.

Keating, P. A. (2006). Phonetic encoding of prosodic structure. In Harrington, J. and Tabain, M., editors, Speech Production: Models, Phonetic Processes and Techniques, Macquarie Monographs in Cognitive Science, chapter 11, pages 167-186. Psychology Press, New York.

Kentner, G. and Féry, C. (2013). A new approach to prosodic grouping. The Linguistic Review, 30(2):277-311.

Klassen, J. and Wagner, M. (2017). Prosodic prominence shifts are anaphoric. Journal of Memory and Language, 92:305-326. 
Klatt, D. H. (1975). Vowel lengthening is syntactically determined in a connected discourse. Journal of Phonetics, 3:129-140.

Krifka, M. (2008). Basic notions of information structure. Acta Linguistica Hungarica, 55(3):243-276.

Kubozono, H. (1989). Syntactic and rhythmic effects on downstep in Japanese. Phonology, 6:39-67.

Kubozono, H. (1992). Modeling syntactic effects on downstep in Japanese. In Docherty, G. and Ladd, R., editors, Papers in laboratory phonology, vol. II: Gesture, segment, prosody, pages 368-287. Cambridge University Press, Cambridge.

Kügler, F. and Féry, C. (2017). Postfocal downstep in German. Language and Speech, 60:260-288.

Kuznetsova, A., Brockhoff, P. B., and Christensen, R. H. B. (2013). Imertest: Tests for random and fixed effects for linear mixed effect models (lmer objects of lme4 package). R package version, pages 2.0-25.

Ladd, D. R. (1980). The Structure of Intonational Meaning. Indiana University Press, Bloomington.

Ladd, D. R. (1988). Declination and 'reset' and the hierarchical organziation of utterances. JASA, 84:530-544.

Ladd, D. R. (2008). Intonational Phonology. Cambridge University Press, Cambridge, 2nd edition. 
Lavoie, L. (2001). Consonant strength: Phonological patterns and phonetic manifestations. Garland, New York.

Lehiste, I. (1973). Phonetic disambigation of syntactic ambiguity. Glossa, 7:107122.

Liberman, M. and Pierrehumbert, J. (1984). Intonational variance under changes in pitch range and length. In Aronoff, M. and Oehrle, R., editors, Language sound structure, pages 157-233. MIT Press, Cambridge, Ma.

Liberman, M. Y. (1975). The Intonational System of English. PhD thesis, MIT.

Martin, James, G. (1970). On judging pauses in spontaneous speech. Journal of Verbal Learning and Verbal, 9:75-78.

Martin, J. (1971). Some acoustic and grammatical features of sponaneous speech. In Horton, D. and Jenkins, J., editors, The perception of language, pages 47-68. C.E. Merril, Columbus, OH.

McAuliffe, M., Socolof, M., Mihuc, S., Wagner, M., and Sonderegger, M. (2017a). Montreal forced aligner: trainable text-speech alignment using Kaldi. In Proceedings of Interspeech 2017 in Stockholm.

McAuliffe, M., Stengel-Eskin, E., Socolof, M., and Sonderegger, M. (2017b). PolyglotDB [software package]. https://github.com/ MontrealCorpusTools/PolyglotDB.

Möbius, B. (1993). Ein quantitatives Modell der deutschen Intonation: Analyse und Synthese von Grundfrequenzverläufen. Walter de Gruyter. 
Mücke, D., Grice, M., and Cho, T. (2014). More than a magic moment-paving the way for dynamics of articulation and prosodic structure. Journal of Phonetics, $44: 1-7$.

Newman, S. S. (1946). On the stress system of English. Word, 2:171-187.

Norcliffe, E. and Jaeger, T. (2005). Accent-free prosodic phrases? Accents and phrasing in the post-nuclear domain. In Proceedings of Interspeech 2005.

Öhmann, S. (1967). Word and sentence intonation: A quantitative model. KTH Department of Speech, Music and Hearing. Quarterly Status Report.

Panayotov, V., Chen, G., Povey, D., and Khudanpur, S. (2015). Librispeech: an ASR corpus based on public domain audio books. In Proc. ICASSP 2015, pages 5206-5210.

Petrone, C., Truckenbrodt, H., Wellmann, C., Holzgrefe-Lang, J., Wartenburger, I., and Höhle, B. (2017). Prosodic boundary cues in german: Evidence from the production and perception of bracketed lists. Journal of Phonetics, 61:71-92.

Pierrehumbert, J. (1979). The perception of fundamental frequency declination. The Journal of the Acoustical Society of America, 66(2):363-369.

Pierrehumbert, J. (1980). The phonology and phonetics of English Intonation. $\mathrm{PhD}$ thesis, MIT.

Pierrehumbert, J. and Hirschberg, J. (1990). The meaning of intonational contours in the interpretation of discourse. In Cohen, P. R., Morgan, J., and Pollack, M. E., editors, Intentions in Communication, pages 271-311. MIT Press, Cambridge, Ma. 
Poschmann, C. and Wagner, M. (2016). Relative clause extraposition and prosody in German. Natural Language E Linguistic Theory, 34(3):1021-1066.

Price, P. J., Ostendorf, M., Shattuck-Hufnagel, S., and Fong, C. (1991). The use of prosody in syntactic disambiguation. Journal of the Acoustical Society of America, 90(6):2956-2970.

Prince, A. S. (1983). Relating to the grid. Linguistic Inquiry, 14:19-100.

Reinhart, T. (2006). Interface Strategies. MIT Press, Cambridge, Ma.

Rietveld, A. C. M. and Gussenhoven, C. (1985). On the relation between pitch excursion size and prominence. Journal of Phonetics, 13(3):299-308.

Rooth, M. (1985). Association with Focus. PhD thesis, University of Massachussetts, Amherst.

Rooth, M. (1992). A theory of focus interpretation. Natural Language Semantics, 1(1):75-116.

Schlöder, J. J. (2018). Assertion and rejection. $\mathrm{PhD}$ thesis, Institute for Logic, Language and Computation, Amsterdam.

Schwarzschild, R. (1999). Givenness, AVOIDF and other constraints on the placement of accent. Natural Language Semantics, 7:141-177.

Selkirk, E. (1986). On derived domains in sentence phonology. Phonology Yearbook, 3:371-405.

Selkirk, E. O. (1984). Phonology and Syntax. The relation between sound and structure. MIT Press, Cambridge, MA. 
Selkirk, E. O. (1995). Sentence prosody: Intonation, stress, and phrasing. In Goldsmith, J. A., editor, Handbook of Phonological Theory, chapter 16, pages 550-569. Blackwell, London.

Shattuck-Hufnagel, S. and Turk, A. E. (1996). A prosody tutorial for investigators of auditory sentence processing. Journal of Psycholinguistic Research, 25(2):193-247.

Streeter, L. A. (1978). Acoustic determinants of phrase boundary perception. The Journal of the Acoustical Society of America, 64(6):1582-1592.

Strobl, C., Boulesteix, A.-L., Kneib, T., Augustin, T., and Zeileis, A. (2008). Conditional variable importance for random forests. BMC Bioinformatics, 9(307).

Strobl, C., Boulesteix, A.-L., Zeileis, A., and Hothorn, T. (2007). Bias in random forest variable importance measures: Illustrations, sources and a solution. $B M C$ Bioinformatics, 8(25).

Strobl, C., Malley, J., and Tutz, G. (2009). An introduction to recursive partitioning: rationale, application, and characteristics of classification and regression trees, bagging, and random forests. Psychological methods, 14(4):323-348.

Sugahara, M. (2003). Downtrends and post-FOCUS intonation in Tokyo Japanese. PhD thesis, University of Massachusetts Amherst, Amherst, MA.

Szendröi, K. (2003). A stress-based approach to the syntax of Hungarian focus. Linguistic Review, 20:37-78.

Tagliamonte, S. A. and Baayen, R. H. (2012). Models, forests, and trees of York 
English: Was/were variation as a case study for statistical practice. Language Variation and Change, 24(2):135-178.

Tao, J., Torreira, F., and Clayards, M. (2018). Durational cues to word boundaries in spontaneous speech. In Proc. 9th International Conference on Speech Prosody 2018, pages 240-244.

t'Hart, J., Collier, R., and Cohen, A. (1990). A perceptual study of intonation: an experimental-phonetic approach to speech melody. Cambridge University Press.

Trouvain, J., Barry, W. J., Nielsen, C., and Andersen, O. (1998). Implications of energy declination for speech synthesis. In The Third ESCA/COCOSDA Workshop (ETRW) on Speech Synthesis.

Truckenbrodt, H. (1995). Phonological phrases: Their relation to syntax, focus, and prominence. $\mathrm{PhD}$ thesis, MIT, Cambridge, Mass.

Truckenbrodt, H. (1999). On the relation between syntactic phrases and phonological phrases. Linguistic Inquiry, 30(2):219-255.

Truckenbrodt, H. (2012). Semantics of intonation. In Von Heusinger, K., Maienborn, C., and Portner, P., editors, Semantics. An international handbook of natural language meaning, volume 3, pages 2039-2969. Mouton De Gruyter, Berlin.

Truckenbrodt, H. and Féry, C. (2015). Hierarchical organisation and tonal scaling. Phonology, 32(01):19-47. 
Turk, A. and Sawusch, J. (1997). The domain of accentual lengthening in American English. Journal of Phonetics, 25(1):25-41.

Turk, A. and Shattuck-Hufnagel, S. (2007). Multiple targets of phrase-final lengthening in American English words. Journal of Phonetics, 35(4):445-472.

Vainio, M. and Järvikivi, J. (2006). Tonal features, intensity, and word order in the perception of prominence. Journal of Phonetics, 34(3):319-342.

Vaissière, J. (1983). Language-independent prosodic features. In Prosody: Models and Measurements, pages 53-65. Springer.

van den Berg, R., Gussenhoven, C., and Rietveld, T. (1992). Downstep in Dutch: implications for a model. In Docherty, G. and Ladd, R., editors, Papers in Laboratory Phonology, vol. II: Gesture, segment, prosody, pages 335-58. Cambridge University Press, Cambridge.

Vander Klok, J., Wagner, M., and Goad, H. (2018). Prosodic focus in English vs. French: A scope account. Glossa: a journal of general linguistics, 3(1)(71):147.

Wagner, M. (2005). Prosody and Recursion. PhD thesis, MIT.

Wagner, M. (2010). Prosody and recursion in coordinate structures and beyond. Natural Language $\mathcal{E}$ Linguistic Theory, 28(1):183-237.

Wagner, P., Cwiek, A., and Samlowski, B. (this special issue). Exploiting the speech-gesture link to capture fine-grained prosodic prominence impressions and listening strategies. Journal of Phonetics. 
Welby, P. (2003). Effects of pitch accent position, type, and status on focus projection. Language and Speech, 46(1):53-81.

Wightman, C. W., Shattuck-Hufnagel, S., Ostendorf, M., and Price, P. J. (1992). Segmental durations in the vicinity of prosodic phrase boundaries. Journal of the Acoustical Society of America, 92:1707-1717.

Williams, E. (1997). Blocking and anaphora. Linguistic Inquiry, 28(4):577-628.

$\mathrm{Xu}$, Y. (2005). Speech melody as articulatorily implemented communicative functions. Speech Communication, 46:220-251.

Xu, Y., Chen, S.-W., and Wang, B. (2012). Prosodic focus with and without postfocus compression: A typological divide within the same language family? The Linguistic Review, 29:131-147. 